\title{
The Quantification of Subjectivity: The R-fuzzy Grey Analysis Framework
}

\author{
Arjab Singh Khuman ${ }^{\mathrm{a}, *}$, Yingjie Yang ${ }^{\mathrm{a}}$, Robert John ${ }^{\mathrm{b}}$ \\ ${ }^{a}$ Institute of Artificial Intelligence (IAI), School of Computer Science and Informatics, De Montfort University, The \\ Gateway, Leicester LE1 9BH, UK \\ ${ }^{b}$ Automated Scheduling, Optimisation and Planning (ASAP), School of Computer Science, Nottingham University, \\ Nottingham NG8 $1 B B$, UK
}

\begin{abstract}
This paper puts forward a newly derived framework for capturing and inferring from subjective based uncertainty for any given observation. The framework is referred to as the R-fuzzy grey analysis framework (RfGAf), which itself is comprised of 3 distinct components: 1. R-fuzzy sets - to capture the uncertainty, which utilises crisp rough set bounding of uncertain possible fuzzy membership values. 2. A significance measure - to provide a means to allow for conditional probability to be undertaken, and also to allow for the translation of the data to that of a time series, allowing for the linking to that of the third component. 3. Grey analysis, more specifically, the absolute degree of grey incidence, where post-analysis can be undertaken and additional metrics obtained. The hybridisation of all three has allowed for the creation of a framework ideally suited for the quantification of perception based uncertainty, which by proxy will be inherently associated to subjectivity. It will be shown and demonstrated how such a framework can be made use of, showcasing the advantages of such an approach. By making use of R-fuzzy sets and the significance measure, an intermediary approach to that of a generalised type-2 fuzzy set can be obtained. As it is widely agreed upon that a generalised type-2 fuzzy approach is ideal for capturing higher degrees of resolution with regards to uncertainty, the associated computational burden of its complexity makes it unfavourable, hence why the interval-valued type-2 approach is favoured. The findings indicate the RfGAf can allow for the high capacity and detail one would expect when considering a type-2 fuzzy set representation, with that of the simplistic objectiveness one would associate to a typical type-1 fuzzy set. The novelty of the framework allows for one to fully capture all the nuances and individualities of a population without a single loss of information. That snapshot in time can tell an awful lot with regards to perceived perception. The framework can be deployed on varying sizes of populations, from the intrinsically small to the overtly large. Potentially, one can use that snapshot to predict how an observation could be perceived in future events. If one can forecast the perception ahead of time, one can improve drastically on efficacy and efficiency. Such a framework has a strong applicability with regards to expert and intelligent systems in allowing for more detailed inference to be utilised and acted upon.
\end{abstract}

Keywords: Subjective modelling, Perception modelling, Fuzzy theory, Grey theory, Uncertainty modelling, Uncertainty theory 


\section{Introduction}

This paper puts together the authors' collective contributions to present a novel framework for capturing subjective based uncertainty. This paper will provide a breakdown of the constituent parts that make-up the framework, so to present to the reader a detailed understanding of its configuration. This will also play a part

5 in providing one with a justification as to why such a framework is needed. It is believed that through the worked examples, the reader will gain an appreciation to the applicability and application of the framework. The work contained in Khuman et al. (2016a) investigated the application of the significance measure used in conjunction with R-fuzzy sets, this created the foundation from which the framework is established. This paper introduces the notion of grey analysis theory, more specifically, the notion of the absolute degree of grey incidence, in conjunction with the significance measure and R-fuzzy sets. The introduction of the grey component completes the framework, ergo, it is this paper that formally presents the newly proposed framework in its entirety; the R-fuzzy grey analysis framework (RfGAf).

To understand the need as to why another model for uncertainty is needed, one needs to consider the sentiment of uncertainty, it becomes quickly apparent that uncertainty should not be generically defined, but rather its definition should be related to the context in which it is involved. As such, there have been, and continue to be new models proposed to handle the various types of uncertainty that exist. Of these models, there are advantages and disadvantages, benefits and shortcomings. As the framework at its foundation is comprised of R-fuzzy sets, one should be made aware of its advantages over the more established models. R-fuzzy was chosen for its ability to capture uncertain fuzzy membership values within a rough set bounding. It is itself an extension to that of fuzzy sets. The R-fuzzy configuration utilises a lower and upper approximation taken directly from roughs set theory, which allows for the membership function of an R-fuzzy set to be that of a rough set. Assuming that a population was asked to provide their perception on a given observation, and using the voting method, any fuzzy membership value contained in the lower approximation would be considered an absolute truth. Whereas, any fuzzy membership value belonging to the upper approximation would be deemed a partial truth. Without the introduction of the significance measure, any value contained in the upper approximation would have no association of strength or popularity. It could be a single instance, or it could have been unanimously voted in favour of, but not by absolutely everyone, this could potentially be a rather large interval of uncertainty in and of itself.

\footnotetext{
*Corresponding author. Tel: +44 (0) 1162506251

Email addresses: Arjab.Khuman@dmu.ac.uk (Arjab Singh Khuman), yyang@dmu.ac.uk (Yingjie Yang), Robert.John@nottingham.ac.uk (Robert John)
} 
This paper puts forward a newly derived framework for capturing subjective based uncertainty - the Rso fuzzy grey analysis framework (RfGAf). It will be described in its entirety as too will its constituent parts, as this will provide the reader with a concrete understanding of the framework and its desired purpose. This paper is an extension to the authors' previous work on extending the notion of R-fuzzy sets (Khuman et al., 2015a) (Khuman et al., 2016a) (Khuman et al., 2016b) (Khuman, 2016). Previously, the authors have introduced the addition of the significance measure to that of R-fuzzy sets, also the notion of grey encapsulation for streamlined derived sets, however, this publication is the first to concretely put together a framework which utilises the authors' collective contributions; bringing the pieces of the jig-saw together.

Khuman enhanced the notion of R-fuzzy sets by putting forward the concept of a significance measure (Khuman et al., 2015a) (Khuman et al., 2016a). The significance measure allowed for conditional probability to be incorporated in relation to all contained values within the membership set of an R-fuzzy set. As all the contained values in the lower approximation are considered absolute truths, their returned degrees of significance would be a 1. The significance measure concerns itself predominately with those values contained in the upper approximation, for these values have an unknown significance in relation to other possible collected candidate values. The returned degrees of significance for these values would, and can only be in the range of greater than 0 , and less than 1 . The exact significance could then be used to determine 45 if the value could be promoted to the lower approximation; a value that was almost voted in favour of with the exception of at least a single voter or some derived threshold of allowance. Or equally, if the value could be demoted to be not contained in the upper approximation; a value that only had a single voter register it as an appropriate indicative value, or by not surpassing some derived threshold for acceptance.

The pairing of R-fuzzy sets and the significance measure provided for an effective means to capture viable fuzzy membership function values for a given observation based on a population. This played its part with regards to providing membership values along with their conditional probability in relation to strength, when precise memberships are not known, a problem that conventional fuzzy based uncertainty models still face. To provide a crisp membership function for a type-1 fuzzy set is not as straight forward as it may seem, there may be issues with vagueness and ambiguity, the plethora of extensions to type-1 fuzzy are all testaments to this very fact. We have the likes of Atanassov intuitionistic fuzzy sets (Atanassov, 1986), where the membership is presented with a degree of non-membership. The notion of shadowed sets was also put forward (Pedrycz, 1998), although this could be seen as a reduction in overall computation as the shadowed set concerned itself with 3 distinct areas of classification, similar semantically to that of the R-fuzzy approach; absolute inclusion, absolute non-inclusion and inclusion to the shadowed region to an unknown degree. Interval-valued fuzzy sets have also been a popular adage (Sambuc, 1975), where the membership of an element is given its membership as an interval, although when considering an interval, uniformed distribution has to be assumed. Even with these new concepts, the use of multiple parameters, intervals and additional fuzzy sets to help describe uncertain fuzzy membership values, would find great 
difficulty in identifying uniqueness in the collected values. This may be an acceptable trade-off, however, as 65 it will be demonstrated, the RfGAf maintains absolute resolution at every stage of its processing; no loss of information or detail is suffered.

The proposed framework has been created to cater for collecting and inferencing from subjective based uncertainty for a given population. As it will be shown through the worked examples, one of the key advantages of the framework is the ability to compare and contrast sub-clusters of cohorts contained in the data. If one can capture the perception of an individual, or cohort indicative of a demographic, then one can use this information to aid and facilitate the creation better perceived observations, whether they are visual, audio, and so on. Knowing how a demographic currently perceives an observation, serves a great purpose is anticipating how they could possibly perceive the same observation, or variance of that observation in the future. Providing this information for an expert or an intelligent system will allow for a system with greater detailed resolution based on higher derived inference.

\subsection{Motivation}

The inspiration behind the framework, is somewhat the same inspiration that inspired the creation of an R-fuzzy set; to be able to better handle uncertain information. The most favourable in terms of the amount of quality, detailed uncertainty that can be captured would have to be that of a type-2 fuzzy set (Mendel and John, 2002), as remarked upon in the abstract. As the membership of a type-2 fuzzy set is a type-1 fuzzy set, there are links to that of R-fuzzy sets, and especially to when R-fuzzy sets are paired with the significance measure, as a significance measure has been proven to be a special instance of a type-1 fuzzy set (Khuman et al., 2015a).

R-fuzzy sets on their own can be seen to be very intuitive, in that they tackle the problem of defining a crisp uncertain membership function for a fuzzy set by providing one with a set of viable possible fuzzy membership values. By making use of a lower and upper approximation, one can reduce the restriction of crispness in terms of providing a single value, but rather a set of possible values, albeit, it is still perfectly possible that an R-fuzzy set could return a single value with regards to its lower approximation. The upper approximation will most likely contain several values, but up until this point those values contained in the upper approximation would have a unified distribution associated to them. The introduction of the significance measure provides one with the facility to quantify the strength of each contained uncertain fuzzy membership value, hence allowing for each value to not necessarily have a uniformed distribution, but rather a varied one. Uniformed distribution would be akin to an interval-valued type-2 fuzzy approach, and non-uniformed distribution would be more in keeping with a generalised type-2 fuzzy approach.

This paper will be given the following structure, as this is the first major effort in describing the RfGAf in its entirety, we will present all the foundational preliminaries in Section 2. This will include the definitions and description of fuzzy sets, rough sets and R-fuzzy sets. Also included will be the significance measure 
and the grey analysis component. Once these have been put forward one will have the understanding needed to appreciate the workings of the RfGAf. Section 3 will demonstrate and describe through worked examples the effectiveness of the proposed framework. It is envisioned that the examples themselves will help better contextualise the applicability of the framework in a plethora of different application areas. There exists several relationships between R-fuzzy sets and the significance measure to that of fuzzy sets, this Section 4 will elaborate on this in more detail, with emphasis to that of type-2 fuzzy sets. Section 5 will present the reader with remarks concerning potential future work and areas of interest. Section 6 will conclude the paper and summaries upon the sentiment.

\section{Framework Preliminaries}

This section will include definitions and descriptions for all attributable aspects of the RfGA framework and areas of interest, presented in a logical order.

\subsection{Fuzzy Set Preliminaries}

As the proposed framework is heavily associated to fuzzy theory, we begin with the notion of fuzzy sets, a fundamental aspect of fuzzy theory and an effective means to capture uncertainty with relation to linguistic phenomena.

Definition 1 (Fuzzy set (Zadeh, 1965)): Let $\mathbb{U}$ represent the universe and let $A$ be a set in $\mathbb{U}(A \subseteq \mathbb{U})$. The fuzzy set $A$ is a set of ordered pairs given by the following expression:

$$
A=\left\{\left\langle x, \mu_{A}(x)\right\rangle \mid x \in \mathbb{U}\right\}
$$

Here, $\mu_{A}: \mathbb{U} \rightarrow[0,1]$ describes the membership function where the returned value $\mu_{A}(x)$ is indicative of the object's degree of membership to the fuzzy set $A$. A returned value of 0 implies no association whatsoever, whereas a returned value of 1 implies total belongingness, and any value in the range $[0,1]$ implies partial belongingness to some degree.

We also have the notion of a type- 2 fuzzy set which itself is an extension to that of a type- 1 fuzzy set. A type-2 fuzzy set makes use of a secondary grade of membership; a fuzzy-fuzzy set so to speak. The secondary grade of membership is type- $(n-1)$, therefore a type-1 fuzzy membership. A generalised type-2 fuzzy approach allows for one to capture a great deal more with regards to uncertainty.

Definition 2 (Type-2 fuzzy set (Mendel and John, 2002)): A type-2 fuzzy set $\tilde{A}$ is characterised by a type-2 membership function $\mu_{\tilde{A}}(x, u)$, where $x \in \mathbb{U}$ and $u \in J_{x} \subseteq[0,1]$. A type-2 fuzzy set is given by the formal expression:

$$
\tilde{A}=\left\{\left\langle(x, u), \mu_{\tilde{A}}(x, u)\right\rangle \mid \forall x \in \mathbb{U}, \forall u \in J_{x} \subseteq[0,1]\right\}
$$


There are two distinct type-2 fuzzy set perspectives; the interval-valued type-2 fuzzy set (IVFS) and that of a generalised type-2 fuzzy set. With regards to an IVFS, all secondary membership values are given a value of 1 , therefore no new information is recorded in relation to the amplitude and the stick heights of the secondary grade objects. Instead, the footprint-of-uncertainty (FOU) is used. The generalised approach allows for every contained secondary grade object to be given a value of membership, however, this is computationally exhaustive, so current type-2 fuzzy approaches will often adopt an interval-valued approach. A concise review of current IVFS applications was undertaken by Melin and Castillo (2014).

When one makes use of multiple fuzzy sets and variations of membership functions, one is then able to provide for a comprehensive encapsulation of any given abstract notion or observation. Such instances involving perception will often involve conflict of opinion; what is warm to some, may not be as warm to others. Depending on the size of the populous, the possible level of conflictions could be rife or minimal. All opinions held are opinions nonetheless and should not be ignored, they should be maintained and preserved. This is what many of the current models have problems with, this point was articulated on by Khuman et al. (2016a). As is the nature of perception based uncertainty, common held assumptions may not always hold true for everyone involved. Individuals are well within their right to provide an opinion that goes against the grain of common held beliefs.

\subsection{Rough Set Preliminaries}

A key component of the RfGA framework is that of rough sets, in this section we provide the reader with an overview of the core concepts that have been made use of. The area of association when concerning oneself or observation, in relation to the attributes belonging to the data it was birthed from. There are 3 distinct areas of classification that an object can belong to; the lower approximation includes all objects that can unequivocally be associated to the set being approximated. The upper approximation includes all objects that have some association to some degree to the set being approximated. The boundary set is therefore 
the difference between the lower and upper approximation spaces. The result of which will determine if the set being approximated is precisely defined or not. There is a fourth category, this is defaulted to absolute non-inclusion, where the object has no association to the set being approximated. Considering the boundary set, the larger the cardinality of this set the greater the imprecision, vagueness and ambiguity that exists for the set being approximated. The fewer objects contained in the boundary set, the less imprecision, vagueness and ambiguity that exists for the set being approximated. It is this notion of approximations that R-fuzzy sets and by proxy what the RfGAf utilises in bounding the uncertain fuzzy membership values.

Rough set theory has been predominately utilised in both decision systems and information systems. An information system is given as a pair; $\Lambda=(\mathbb{U}, A)$, where $\mathbb{U}$ is a non-empty finite set of objects referred to as the universe. $A$ is a non-empty finite set of attributes such that $a: \mathbb{U} \rightarrow V_{a}$ for every $a \in A$. The set $V_{a}$ is the value set for $a$. If the data set represents that of a decision system, then the notation would make use of the decision attribute; $\Lambda=(\mathbb{U}, A \cup\{d\})$, where $d \notin A$. Depending of the size of the data from which the system is based, one may be faced with superfluous information. Making use of the equivalence and indiscernibility relation can be undertaken to reduce this burden. Assume a binary relation $R \subseteq X \times X$ exists, where $R$ is referred to as the equivalence relation. The equivalence class of an element $x \in X$ contains all objects $y \in X$ such that $x R y$.

Definition 3 (Equivalence relation (Pawlak, 1998, 2002)): Assume that $\Lambda=(\mathbb{U}, A)$ is an information system and with any $B \subseteq A$ there is an associated equivalence relation $\operatorname{IND}_{\Lambda}(B)$, given by the formal expression:

$$
\operatorname{IND}_{\Lambda}(B)=\left\{\left(x, x^{\prime}\right) \in \mathbb{U}^{2} \mid \forall a \in X a(x)=a\left(x^{\prime}\right)\right\}
$$

Here, $\operatorname{IND}_{\Lambda}(B)$ is the $B$-indiscernibility relation. If $\left(x, x^{\prime}\right) \in \operatorname{IND}_{\Lambda}(B)$, then it can be concluded that both $x$ and $x^{\prime}$ are indeed indiscernible from one another by the attributes that are available in $B$.

Definition 4 (Approximations (Pawlak, 1998, 2002)): Assume that $\Lambda=(\mathbb{U}, A)$ is an information system and that $B \subseteq A$ and $X \subseteq \mathbb{U}$. One can approximate set $X$ with the information contained in $B$ via a lower and upper approximation set.

The lower approximation is described as the set containing all objects that unequivocally belong to the set $X$ in relation to $B$. Given by the following expression:

$$
\begin{gathered}
\underline{B} X=\left\{x \mid[x]_{B} \subseteq X\right\} \\
\underline{B}(x)=\bigcup_{x \in \mathbb{U}}\{B(x): B(x) \subseteq X\}
\end{gathered}
$$

The upper approximation is described as the set containing all possible objects that have an association to the set $X$ in relation to $B$. Given by the following expression:

$$
\bar{B} X=\left\{x \mid[x]_{B} \cap X \neq \emptyset\right\}
$$




$$
\bar{B}(x)=\bigcup_{x \in \mathbb{U}}\{B(x): B(x) \cap X \neq \emptyset\}
$$

The boundary set is described as being the difference of the upper and lower approximations. Given by the following expression:

$$
B N(X)=\bar{B}(X)-\underline{B}(X)
$$

Definition 5 (Rough sets $(Y a o, 1996))$ : Assume that the pair, apr $=(\mathbb{U}, B)$ is an approximation space on $\mathbb{U}$ and assume that $\mathbb{U} / B$ denotes the set of all equivalence classes over $B$. The family of all definable sets in approximation space apr is denoted by $\operatorname{def}(a p r)$. Given two subsets $\underline{A}, \bar{A} \in \operatorname{def}($ apr $)$ with $\underline{A} \subseteq \bar{A}$, the pair $(\underline{A}, \bar{A})$ is called a rough set.

- if $x \in \underline{A}$ then $x \in(\underline{A}, \bar{A})$

- if $x \in \mathbb{U}-\bar{A}$ then $x \notin A$

- if $x \in \bar{A}$ and $x \notin \underline{A}$ then $x$ has an unknown relation to $(\underline{A}, \bar{A})$

If the boundary region of the approximated set is empty, then the approximated set is crisp. If the boundary region of the approximated set is non-empty, then the approximated set is indeed rough.

\subsection{R-Fuzzy Set Preliminaries}

We now present the main aspect of the proposed frame work, the notion of an R-fuzzy set. A method for encapsulating uncertain fuzzy membership values in a rough set membership function, via the approximations defined in Definition 4.

Definition 6 (An R-fuzzy set (Yang and Hinde, 2010)): Let the pair apr $=\left(J_{x}, B\right)$ be an approximation space on a set of values $J_{x}=\left\{v_{1}, v_{2}, \ldots, v_{n}\right\} \subseteq[0,1]$, and let $J_{x} / B$ denote the set of all equivalence classes of $B$. Let $\left(\underline{M}_{A}(x), \bar{M}_{A}(x)\right)$ be a rough set in apr. An R-fuzzy set $A$ is characterised by a rough set as its membership function $\left(\underline{M}_{A}(x), \bar{M}_{A}(x)\right)$, where $x \in \mathbb{U}$, given by the formal expression:

$$
A=\left\{\left\langle x,\left(\underline{M}_{A}(x), \bar{M}_{A}(x)\right)\right\rangle \mid \forall x \in \mathbb{U}, \underline{M}_{A}(x) \subseteq \bar{M}_{A}(x) \subseteq J_{x}\right\}
$$

When one considers the likes of type-2 fuzzy sets as presented in Definition 2, and interval-valued fuzzy sets, the similarities to that of an R-fuzzy set become clear, as they all essentially use a set to describe its membership function, although an interval-valued approach will make use of an interval rather than a set per se. If there are uncertain fuzzy values that have an affinity to an R-fuzzy descriptor, then said uncertain fuzzy value will be included in the membership set.

For each $x_{i} \in \mathbb{U}$, there is an associated membership description $d\left(x_{i}\right)$ which describes the belongingness of the object for consideration $x_{i}$ to the set $A \subseteq \mathbb{U}$. A population of candidates whom provide their subjective 
perceptions are collected and contained in $C$, the set of available evaluation criteria. After which, each value $v \in J_{x}$ is evaluated by $c_{j} \in C$ to establish if it is described by the membership description for $x_{i}$ with respect to $A$. The result of the evaluation is given by an unequivocal YES or NO. Evaluations which result in a YES are forwarded and evaluations which result in a NO are simply ignored.

$$
v \stackrel{\left(d\left(x_{i}\right), c_{j}\right)}{\longrightarrow} \text { YES } \quad \text { or } \quad v \stackrel{\left(d\left(x_{i}\right), c_{j}\right)}{\longrightarrow} \mathrm{NO}
$$

For each pair $\left(\left(x_{i}\right), c_{j}\right)$ where $x_{i} \in \mathbb{U}$ and $c_{j} \in C$, a set $M_{c j}\left(x_{i}\right) \subseteq J_{x}$ is created, given by the formal expression:

$$
M_{c j}\left(x_{i}\right)=\left\{v \mid v \in J_{x}, v \stackrel{\left(d\left(x_{i}\right), c_{j}\right)}{\longrightarrow} \mathrm{YES}\right\}
$$

The lower approximation based for the rough set $M\left(x_{i}\right)$ for the membership function given by $d\left(x_{i}\right)$, is given as follows:

$$
\underline{M}\left(x_{i}\right)=\bigcap_{j} M_{c j}\left(x_{i}\right)
$$

The upper approximation based for the rough set $M\left(x_{i}\right)$ for the membership function given by $d\left(x_{i}\right)$, is given as follows:

$$
\bar{M}\left(x_{i}\right)=\bigcup_{j} M_{c j}\left(x_{i}\right)
$$

The rough set approximating the membership is therefore given as:

$$
M\left(x_{i}\right)=\left(\bigcap_{j} M_{c j}\left(x_{i}\right), \bigcup_{j} M_{c j}\left(x_{i}\right)\right)
$$

\subsection{Significance Measure Preliminaries}

The significance measure was first presented by Khuman et al. (2015a) and plays a pivotal role in the proposed framework, for it is the significance measure that links R-fuzzy sets to that of grey analysis, in particular the absolute degree of grey incidence. The inspiration behind the significance measure is with regard to the certainty factor adopted from traditional rough set theory. The significance measure is itself based on Bayes' theorem and provides one with the conditional probability of each captured uncertainty fuzzy membership value contained in the membership set of each generated R-fuzzy set.

Definition 7 (Significance measure Khuman et al. (2015a)): Using the same notation presented in Definition 6 that described an R-fuzzy set, one has to assume that an R-fuzzy set $M\left(x_{i}\right)$ has already been created, and that a membership set of values $J_{x}$ and a criteria set $C$ are known. Assume that the total number of subsets generated for a given R-fuzzy set is given by $|N|$, and that $S_{v}$ is the number of subsets that contain the specified membership value being inspected. As each value $v \in J_{x}$ is evaluated by $c_{j} \in C$, 
the significance measure therefore counts the number of instances that $v$ occurred over $|N|$, given by the formal expression:

$$
\gamma_{\bar{A}}\{v\}=\frac{S_{v}}{|N|}
$$

It can be seen that the significance measure provides one with the conditional probability that $v \in J_{x}$ belongs to the R-fuzzy set $M\left(x_{i}\right)$, given by its descriptor $d\left(x_{i}\right)$. If the returned value of $\gamma_{\bar{A}}\{v\}=1$, then one can conclude that the membership value has been agreed upon by all in the criteria set $C$, and wholeheartedly belongs in the lower approximation:

$$
\underline{M}_{A}=\left\{\gamma_{\bar{A}}\{v\}=1 \mid v \in J_{x} \subseteq[0,1]\right\}
$$

For all membership values contained in the lower approximation of an R-fuzzy set, the resulting significance measure will be a 1 , this verifies that all collected lower approximation values were indeed agreed upon by all in the criteria set $C$. The significance measure was derived with the upper approximation in mind, as it was this aspect of R-fuzzy set theory that needed enhancing, providing one with the ability to quantify the distribution of collected membership values.

As Definition 6 stipulates, the lower approximation is a subset of the upper approximation, therefore, for every uncertain fuzzy membership contained in the lower approximation, one can absolutely expect to see the same uncertain fuzzy membership value to be contained in the upper approximation. If the returned significance value is $\gamma_{\bar{A}}\{v\}=0$, it can be concluded with absolute certainty that not a single voter perceived that particular value to be indicative of the R-fuzzy descriptor. If however, the returned significance value is $0<\gamma_{\bar{A}}\{v\}<1$, then one will be able to conclude for that particular membership value that is has an affinity to the R-fuzzy set descriptor, and as such will be included in the upper approximation:

$$
\bar{M}_{A}=\left\{\gamma_{\bar{A}}\{v\}>0 \mid v \in J_{x} \subseteq[0,1]\right\}
$$

The aforementioned interpretations are very similar and aligned with that of the fuzzy set as described in Definition 1. Whereby a membership function describes the degree of belongingness to the set. The difference to that of the significance measure is that the significance measure provides one with the measure of significance with regard to the R-fuzzy descriptor, based upon the conditional probability of the inspected value. Therefore, Eq. (12) can be rewritten so that the collected degrees of significance populate a set, presented using the following expression

$$
\bar{A}=\left\{\left\langle v, \gamma_{\bar{A}}\{v\}\right\rangle \mid v \in J_{x} \subseteq[0,1]\right\}
$$

Where $\bar{A}$ is the set describing the distribution of the selected R-fuzzy descriptor $d(x)$.

\subsection{Grey Analysis}

The additional post-analysis attribute of the framework makes use of grey theory, in particular, it utilises the absolute degree of grey incidence from the family of grey analysis operators. It should be noted that 
there are several variations to the degree of grey incidence, but it is the absolute degree of grey incidence that is employed as it compares sequences in their absolute entirety.

What should be understood firstly, is that the use of the absolute degree of grey incidence is undertaken in an unconventional manner. The notion of grey incidence for sequence comparability was first investigated by the authors in (Khuman et al., 2015b), where it was executed in a natural language processing domain. This provided the foundation from which to further expand upon, and ergo the introduction of grey analysis into the enhanced R-fuzzy framework. The traditional use of grey incidence as it was originally envisioned, involves the comparing of a characteristic sequence to that of several behavioral factors, to ascertain how much a particular behaviour influences a selected characteristic. The values are collected and presented in a matrix, $\Gamma=\left[\gamma_{i j}\right]$, one will be able to infer from the matrix how similar or dissimilar a particular sequence is when compared to that of another. The stronger the similarities, the closer the degree of incidence will be to 1 , the more divergence that exists between two comparable sequence the closer the value will be to zero. If the data that the RfGA framework is involved with contains attributes that can further categorise the data contained; sex, nationality, age groups and the like, then the R-fuzzy sets generated from these subsets can be compared and contrasted to investigate divergence in perceptions for any given observation. This will be further explored through the examples which are to follow in the next section.

Definition 8 (Absolute degree of grey incidence Sifeng et al. (2011)Liu and Lin (2006)): Assume that $X_{i}$ and $X_{j} \in \mathbb{U}$ are two sequences of data with the same magnitude, that are defined as the sum of the distances between two consecutive time points, whose zero starting points have already been computed:

$$
\begin{gathered}
s_{i}=\int_{1}^{n}\left(X_{i}-x_{i}(1)\right) d t \\
s_{i}-s_{j}=\int_{1}^{n}\left(X_{i}^{0}-X_{j}^{0}\right) d t
\end{gathered}
$$

Therefore the absolute degree of incidence is given as:

$$
\epsilon_{i j}=\frac{1+\left|s_{i}\right|+\left|s_{j}\right|}{1+\left|s_{i}\right|+\left|s_{j}\right|+\left|s_{i}-s_{j}\right|}
$$

What has been presented in this section are the component parts that constitute the proposed RfGA framework. What is to follow is a demonstration of the proposed framework, with the expectation that reader will gain a more thorough understanding of the framework's capabilities. It is envisioned that the via the examples that will be put forward, one will be able to garner an appreciation as to the importance of such a framework given the implicit and intrinsic nature of perception based uncertainty. 


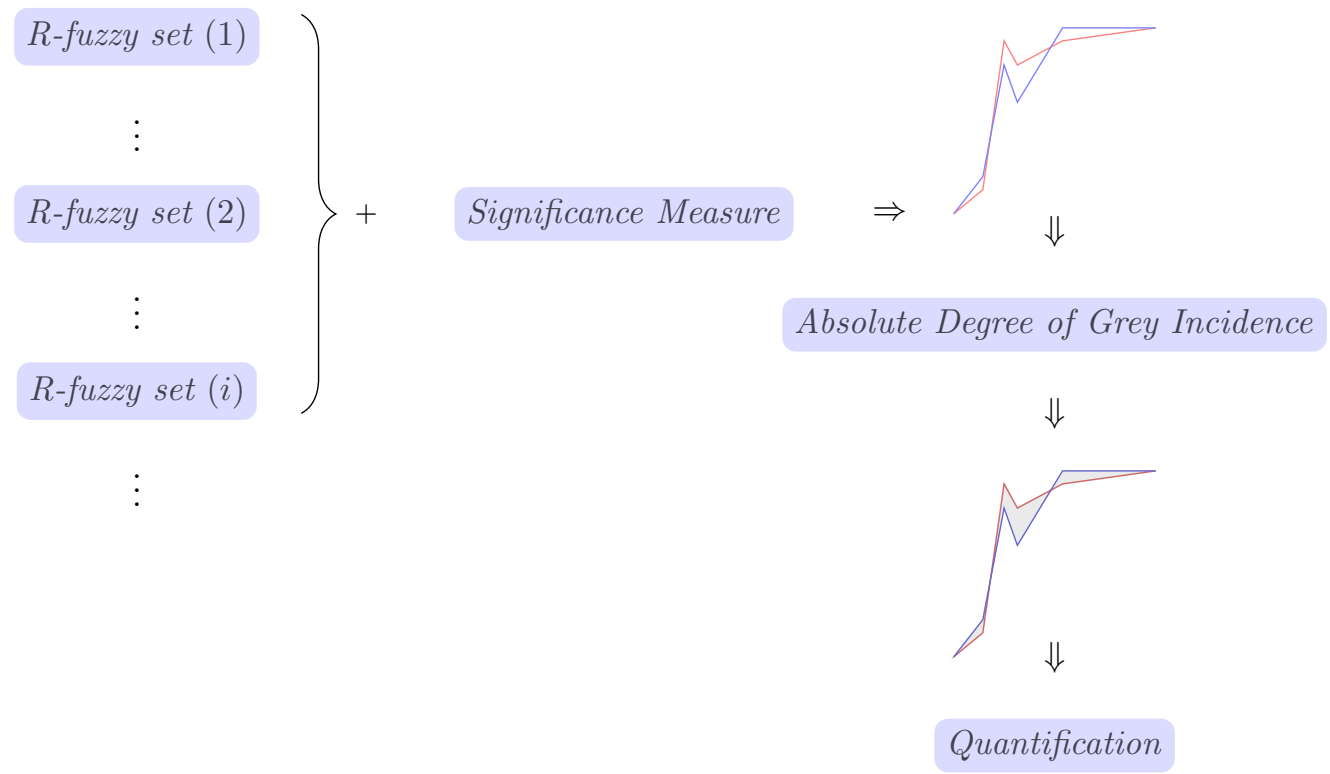

Figure 1: A visualisation of the R-fuzzy grey analysis framework

\section{The R-fuzzy Grey Analysis Framework}

With the essential constituent parts of the proposed framework given, it is now time to demonstrate the approach using worked examples. The examples themselves use real-world data and subjects. As the nature of perception based uncertainty involves the collection of subjectivity, certain consideration has been given to only collect the information needed for the RfGA framework to be affective. As it will be seen, there is versatility and a great deal of robustness associated to the framework, which adds to its appeal. The examples will highlight specific aspects of the framework. To begin, the R-fuzzy and significance measure pairing will be demonstrated, from this, the RfGA framework will then be showcased making use of post grey analysis.

A visualisation of the framework can be seen in Figure 1. The successive R-fuzzy sets when passed to the significance measure allows for the sequences to be generated, from which the absolute degree of grey incidence can then be undertaken. One could simply stop at the significance measure stage and use the information obtained at this point to inspect the consensus of the cohort, but owing to the versatility of the framework, one could continue to garner further levels of inference by utilising the absolute degree of grey incidence.

Example 1: Consider the following set $F=\left\{f_{1}, f_{2}, \ldots, f_{9}\right\}$, which contains the information regarding 9 variations of the colour red. The swatches are given using [RGB] values, although this could easily be 
presented using other arbitrary presentations.

$$
\begin{array}{rlrl}
f_{1} & \rightarrow & {[204,0,0]} & \rightarrow \\
f_{2} & \rightarrow & {[153,0,0]} & \rightarrow \\
f_{3} \rightarrow & {[255,102,102]} & \rightarrow \\
f_{4} & \rightarrow & {[51,0,0]} & \rightarrow \\
f_{5} & \rightarrow & {[255,153,153]} & \rightarrow \\
f_{6} & \rightarrow & {[102,0,0]} & \rightarrow \\
f_{7} & \rightarrow & {[255,204,204]} & \rightarrow \\
f_{8} & \rightarrow & {[255,0,0]} & \rightarrow \\
f_{9} & \rightarrow & {[255,51,51]} & \rightarrow
\end{array}
$$

\begin{tabular}{|c|c|c|c|c|c|c|c|c|c|}
\hline & $f_{1}$ & $f_{2}$ & $f_{3}$ & $f_{4}$ & $f_{5}$ & $f_{6}$ & $f_{7}$ & $f_{8}$ & $f_{9}$ \\
\hline$p_{1}$ & $D R$ & $D R$ & $L R$ & $D R$ & $L R$ & $D R$ & $L R$ & $R$ & $R$ \\
\hline$p_{2}$ & $R$ & $D R$ & $R$ & $D R$ & $L R$ & $D R$ & $L R$ & $R$ & $R$ \\
\hline$p_{3}$ & $R$ & $D R$ & $L R$ & $D R$ & $L R$ & $D R$ & $L R$ & $R$ & $L R$ \\
\hline$p_{4}$ & $D R$ & $R$ & $L R$ & $D R$ & $L R$ & $D R$ & $L R$ & $R$ & $L R$ \\
\hline$p_{5}$ & $D R$ & $D R$ & $R$ & $D R$ & $L R$ & $D R$ & $L R$ & $R$ & $L R$ \\
\hline$p_{6}$ & $D R$ & $D R$ & $L R$ & $D R$ & $L R$ & $D R$ & $L R$ & $R$ & $R$ \\
\hline
\end{tabular}

If we take the average of the $[\mathrm{RGB}]$ values, and place them in a set, we are presented with the following information: $N=\{68,51,153,17,187,34,221,85,119\}$. The index of $N_{i}$ can be easily correlated to its respective swatch from $F_{i}$. For example the average for the swatch given in $f_{7}$ is 221 , as its [RGB] values are given as $[255,204,204]$. Consider a population size of 6 individuals, whom gave their subjective understanding of the swatches contained in $F$, these perceptions have been collected and are presented in Table 1 . The criteria set $C=\left\{p_{1}, p_{2}, \ldots, p_{6}\right\}$ is a fundamental attribute of the framework. The abbreviations contained within the table are translated to the following:

$$
D R \rightarrow \text { Dark Red } \quad R \rightarrow \text { Red } \quad L R \rightarrow \text { Light Red }
$$

At this point we have the foundational underpinnings of the framework populated; we have the colour swatches and their respective representations, in this case their averages; we have a criteria set $C$ populated 
with subjective perceptions for the red swatches. From this, we can generate the fuzzy membership set $J_{x}$ where all associated uncertain fuzzy membership values will be derived. Using the following linear function:

$$
\mu\left(f_{i}\right)=\frac{l_{i}-l_{\min }}{l_{\max }-l_{\min }}
$$

Which results in the following fuzzy membership set:

$$
J_{x}=\{0.25,0.17,0.67,0.00,0.83,0.08,1.00,0.33,0.50\}
$$

Where the advantage of R-fuzzy sets comes into fruition, is with regards to not knowing. We may not know the precise value for a newly presented $10^{t h}$ colour, we may just be presented with the swatch itself. The R-fuzzy notion allows for one to express a possible fuzzy membership function if information is missing, for example if the colour swatch values are not available. Assume that a new colour swatch based on a variation of red is considered and given a descriptor of Red $(R)$, the question now becomes what uncertain fuzzy membership values would one assign this new swatch? If we were to consider the use of a typical type-1 fuzzy set, then a crisp single value would have to be nominated, however, simply inspecting the perceptions contained in Table 1, one can infer that not everyone will perceive the same observation the same way. Therefore using a single value to be indicative of a perception may not be wise as this does not take into account subjectivity. An R-fuzzy set is comprised of a lower and upper approximation, where all contained values in the lower approximation have been agreed upon by all in the criteria set $C$ as being a valid representation. All the values contained in the upper approximation will be indicative of individual objectiveness. Therefore, all contained uncertain fuzzy membership values belonging to an R-fuzzy set will collectively present the consensus held by the entire population and also individual interpretations, all within the same set.

Given that the new $10^{\text {th }}$ colour swatch has an associated descriptor of $R$, and based on the information collected in Table 1, for any value that has an association to the descriptor, will qualify as a valid fuzzy membership value to represent it. For each member of the criteria set $C$, each $p_{i}$ is inspected, $v \in J_{x}$ is evaluated against $p_{j} \in C$ to confirm whether or not it satisfies the descriptor, using the following expression:

$$
v \stackrel{\left(d\left(x_{i}\right), c_{j}\right)}{\longrightarrow} \text { YES }
$$

Inspecting each individual $p_{i}$ in the criteria set $C$, where there is a match with the descriptor of interest, the correlating $f_{i}$ membership value is recorded. For the above example, the descriptor for the newly introduced swatch $f_{10}$ has been given as Red, using the perceptions collected in Table 1 we can now generate the subsets that will collectively contribute to the final R-fuzzy set. Consider the perceptions recorded for the individual at $p_{1}$, one can see that when the descriptor is set to $d\left(f_{10}\right)=$ ' Red', only the colour swatches at $f_{8}$ and $f_{9}$ will register as a match. Therefore, the associated fuzzy membership values in $J_{x}$ for these 
swatches are carried forward. The subset generated from this for $p_{1}$ is given as $M_{p 1}\left(f_{10}\right)=\{0.33,0.50\}$. This will be repeated for the total number of contained individuals in the criteria set $C$, the generated subsets for the remaining individuals are given as:

$$
\begin{array}{ll}
M_{p 1}\left(f_{10}\right)=\{0.33,0.50\} & M_{p 2}\left(f_{10}\right)=\{0.25,0.33,0.50,0.67\} \\
M_{p 3}\left(f_{10}\right)=\{0.25,0.33\} & M_{p 4}\left(f_{10}\right)=\{0.17,0.33\} \\
M_{p 5}\left(f_{10}\right)=\{0.33,0.67\} & M_{p 6}\left(f_{10}\right)=\{0.33,0.50\}
\end{array}
$$

Once all possible subsets have been generated, the approximations as described in Definition 4 can now be applied. If one begins with the lower approximation presented in Eq. (9), each subset is investigated to see which uncertain fuzzy membership value has occurred in all presented subsets. It is clear to see that in our case the only value to be included in each subset is that of 0.33 , and it satisfies the lower approximation significance measure restriction given by Eq. (13). This value is the only value to be contained in the lower approximation set, as there were no other candidates. It is perfectly plausible for the final generated R-fuzzy set to have an empty lower approximation set, if no single fuzzy membership value occurred in all subsets. If one now moves onto the upper approximation as presented in Eq. (10), one can now populate the set with fuzzy membership values that satisfy the upper approximation significance measure restriction given by Eq. (14). Which will include all fuzzy membership values that have been voted by at least one member of the populous, but not everyone, which duplicated values removed: $\{0.17,0.25,0.33,0.50,0.67\}$.

The final R-fuzzy set produced when considering the fuzzy membership values for $d\left(f_{10}\right)$ is presented using Eq. (11), the result of which is as follows:

$$
M\left(f_{10}\right)=(\{0.33\},\{0.17,0.25,0.33,0.50,0.67\})
$$

Inspecting the final concluding set, one can clearly see that the only value to be included in the lower approximation is 0.33 , therefore this was the only value that was deemed suitable to be used when describing the descriptor when set to Red. Draw your attention back to Table 1, one can see that the only colour swatch which was unanimously agreed upon as being $R e d$ was that of swatch $f_{8}$. This swatch itself correlates directly with the $[\mathrm{RGB}]$ of $[255,0,0]$, which when considering the digital representation of what one would deem to be red, in all intents and purposes, is indeed red. The lower approximation is clear cut and absolute, where the notion of variance comes into play is with that of the upper approximation. Consider the following fuzzy membership values $0.17,0.25,0.50$ and 0.67 , which were voted for by the populous to also be possible candidates to represent the colour Red. It is here where the significance measure provides its strength, as it allows for one to truly ascertain the significance of each and every contained uncertain fuzzy membership 


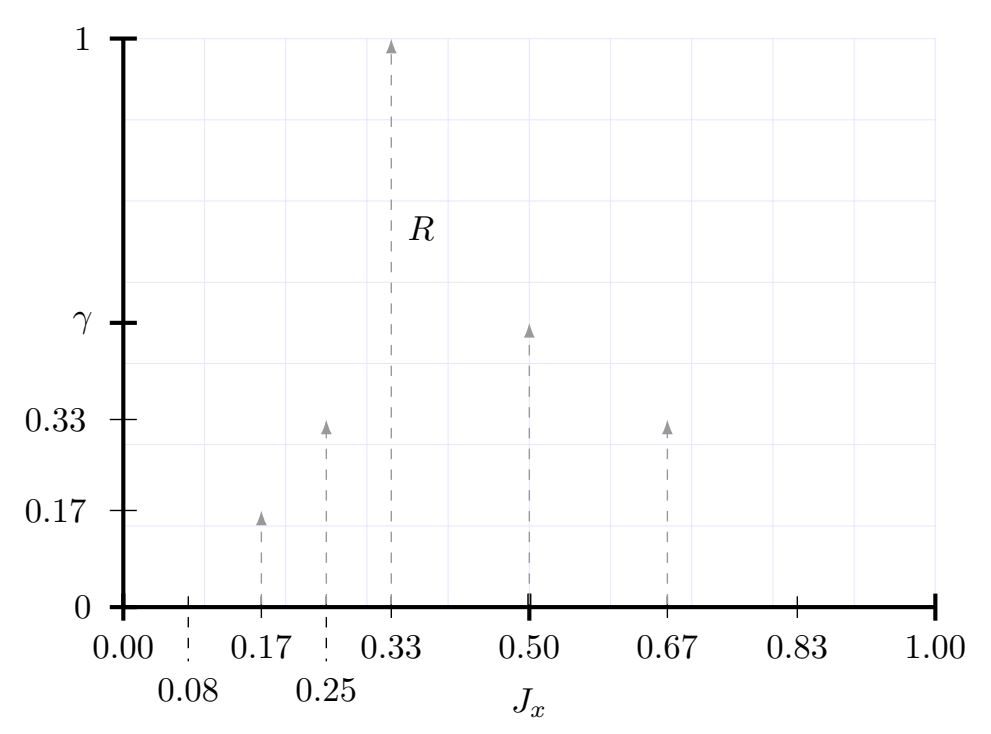

Figure 2: A discrete visualisation for Red, based on the returned degrees of significance

value. Using Eq. (12), the returned degree of significance directly relates itself to the conditional probability of how strong a particular value has been deemed to be, based on all respected votes for all generated subsets. Therefore, the resulting degrees of significance based on the populous contained in Table 1 for when the descriptor is set to $d\left(f_{10}\right)=$ 'Red', is given as follows:

$$
\begin{array}{ll}
\gamma_{\bar{R}}\{0.00\}=\frac{0}{6}=0.00 & \gamma_{\bar{R}}\{0.08\}=\frac{0}{6}=0.00 \\
\gamma_{\bar{R}}\{0.17\}=\frac{1}{6}=0.17 & \gamma_{\bar{R}}\{0.25\}=\frac{2}{6}=\frac{1}{3}=0.33 \\
\gamma_{\bar{R}}\{0.33\}=\frac{6}{6}=1.00 & \gamma_{\bar{R}}\{0.50\}=\frac{3}{6}=\frac{1}{2}=0.50 \\
\gamma_{\bar{R}}\{0.67\}=\frac{2}{6}=\frac{1}{3}=0.33 & \gamma_{\bar{R}}\{0.83\}=\frac{0}{6}=0.00 \\
\gamma_{\bar{R}}\{1.00\}=\frac{0}{6}=0.00 &
\end{array}
$$

The plot contained in Figure 2 is the discrete representation for when the descriptor is set to $d\left(f_{10}\right)=$ 'Red', taking into account all generated degrees of significance. As we know that the only value to be contained in the lower approximation was that of 0.33 , this according to the lower approximation restriction given in Eq. (13) would result in a value of 1 for its significance, and indeed the plot clearly illustrates this. This was always to be expected as the lower approximation deals with absoluteness, it is more telling when 


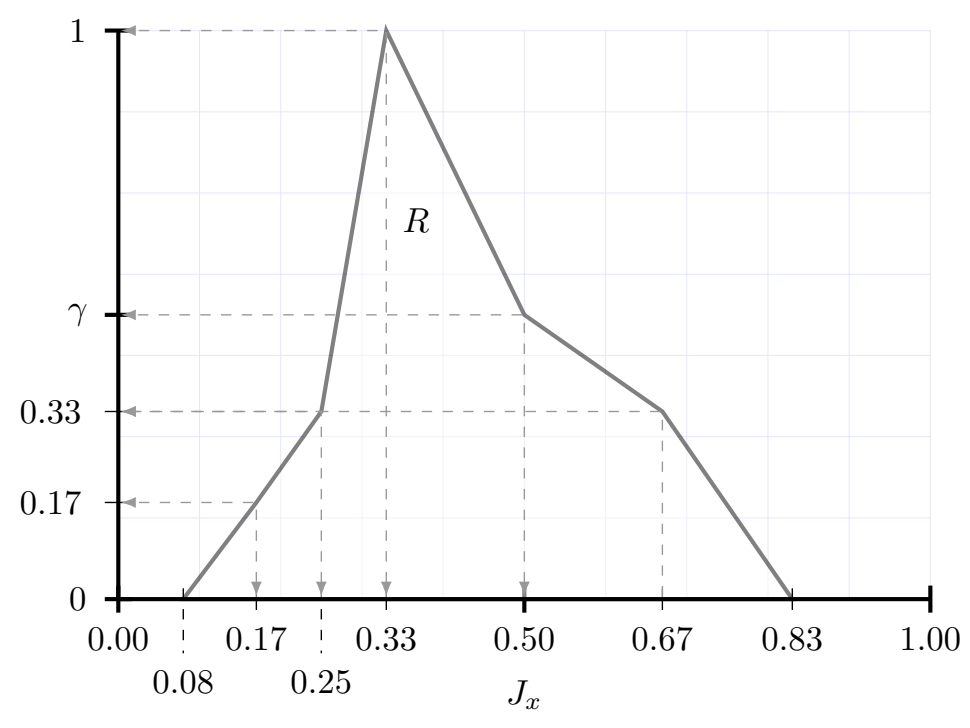

Figure 3: A derivable continuous visualisation for Red, based on the returned degrees of significance

one considers the values that were contained in the upper approximation. Inspecting the returned degrees of significance for these values, and inspecting the plot provides one a more detailed understanding, as one can see that values either side of 0.33 scored relatively high and tapered off the further away from 0.33 . For the fuzzy membership values that were not voted in favour of, they simply return a significance of 0 , and as a result, there is no registered amplitude for their position on the plot.

If one was to draw a line from each fuzzy membership value, to its corresponding degree of significance, one would then create a hull which encapsulates and provides for a continuous representation of the R-fuzzy set for Red, this may not necessarily be a convex hull as will be seen. This can be seen in Figure 3, where a derivable plot is displayed. It is noteworthy to mention the equivalence to that of a type- 1 fuzzy set as defined in Definition 1. The returned degrees of significance act in much the same way as the returned degrees of membership from a fuzzy perspective.

Figure 3 provides one with a derivable continuous representation, however, one could easily apply arbitrary assumptions to the plot if desired. Such an example can be seen in Figure 4, where a clear distinction from the previous plot can be seen. The most noticeable difference is that of the choice of membership function, whereas the plot in Figure 3 made use of what predominately was a triangular membership, Figure 4 utilises more of a trapezoidal membership. It would seem that having a plateau around the apex for the values that scored a 1 for their returned degrees of significance would be more akin to a humanistic and forgiving approach. Since the value 0.33 returned an absolute degree of significance of 1 , it stands to reason that if this was used as a template, and on an unquestioned criteria set, then it would be likely that 0.32 and 0.34 would also score a degree of significance of 1 , or in the very least a very high value, before it tapers off. At what point should the plateau begin to taper and reduce in significance is purely an arbitrary 


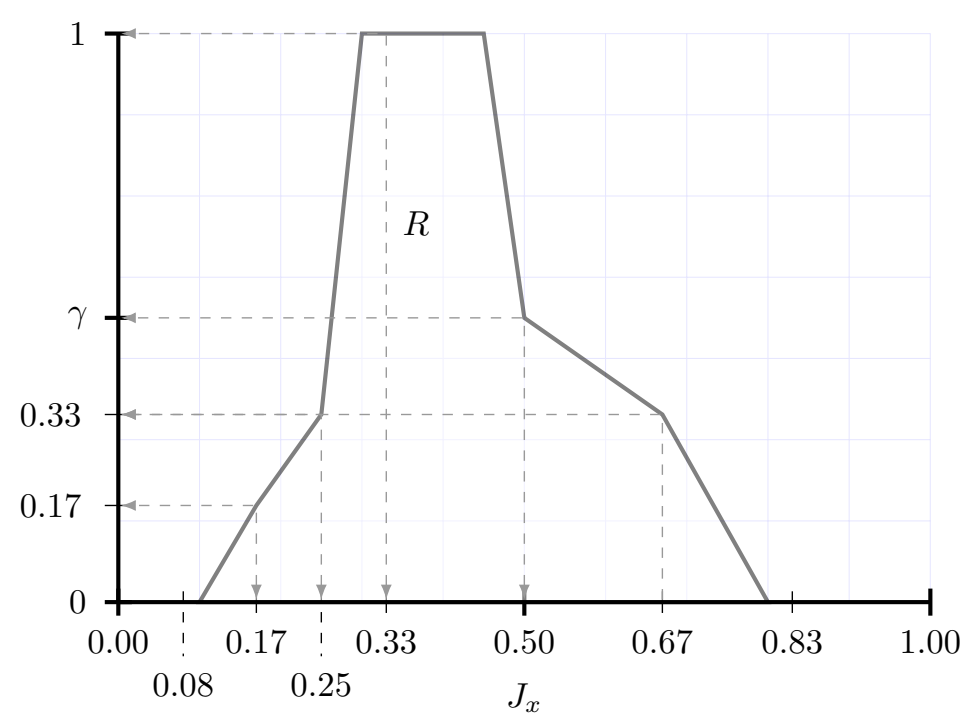

Figure 4: A possible continuous visualisation for Red, based on the returned degrees of significance

assumption, in Figure 4 the plateau covers the interval $[0.3,0.45]$, hence why it is described as a possible interpretation.

Considering the R-fuzzy set Red, if one was to use a standard type-1 fuzzy style approach, and to make use of a singleton value to represent Red, one maybe inclined to use the average, in which case the returned result would be:

$$
\mu\left(f_{10}\right)=\frac{1}{14} \sum_{x \in \mathbb{U}} \mu(x) / x=0.39
$$

This value has been obtained when considering all generated subsets $M_{p j}\left(f_{10}\right) \in J_{x}$ and their contained

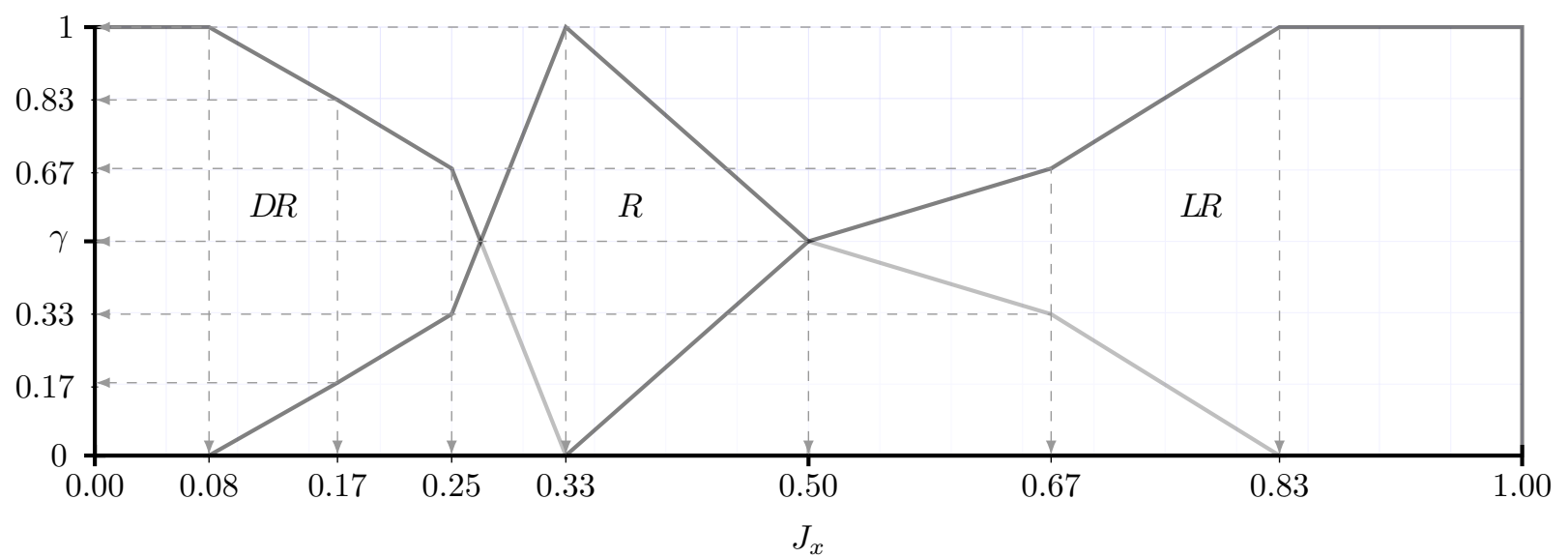

Figure 5: A derivable continuous visualisation for Example 1, based on all the generated significance measures for the R-fuzzy sets of Dark Red, Red \& Light Red 
fuzzy membership values. It is clear to see that the type- 1 fuzzy averaged produced value of 0.39 is considerably greater then the R-fuzzy derived 0.33. The fuzzy produced value would be too skewed and provide for an unrealistic interpretation of the results. If one was to make use of an interval approach, where the left and right most bounds of the interval were the left most pessimistic and the right most optimistic anchor points, one would be presented with an interval too large $[0.17,0.67]$. This would make it impossible to identify which fuzzy membership values would have been agreed upon by all. This is also the same hindrance that would face an Atanassov intuitionistic fuzzy set. As remarked upon earlier in the paper, the current approaches, extensions and hybridisations, find it increasingly difficult to isolate and identify objects contained in intervals and shadowed regions.

The plot presented in Figure 5, provides for a derivable representation of all three R-fuzzy descriptors utilised in Example 1. As one propagates from one R-fuzzy set to its neighbour, all collected and encapsulated returned degrees of significance are conserved. Consider the fuzzy membership value of 0.17 , this returns a

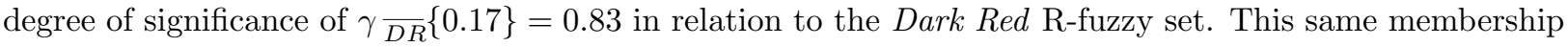
value returns a degree of significance of $\gamma_{\bar{R}}\{0.17\}=0.17$ in relation to the Red R-fuzzy set. In much the same way an observation can be perceived in a multitude of different ways, an uncertain fuzzy membership value can also be reacted to differently depending on the descriptor it is being modelled for. The plot also plays a part in providing an understanding and a visualisation of the populous as a whole. If this was to be indicative of a demographic, one would be able to gauge future responses using this as a template, which could be refined with additional data, in particular more representation on the criteria set $C$. As is the nature of human subjectivity, the area of distribution for each generated R-fuzzy set does not adhere to symmetry, nor is there uniformed distribution. An R-fuzzy approach is more in keeping with a humanistic interpretation, whereas a strictly defined symmetrical encapsulating set would prove too restrictive. Figure 6 provides one with an arbitrary configuration for the distribution of R-fuzzy sets based on that of Figure 5 .

Example 1 demonstrated the pairing of the R-fuzzy and significance measure, as this paper puts forward the notion of the R-fuzzy and grey analysis framework, Example 2 will now go onto demonstrate how the addition of the grey analysis component can be utilised into the framework to create a tool that is far superior in maintaining and inferencing from subjectivity. 


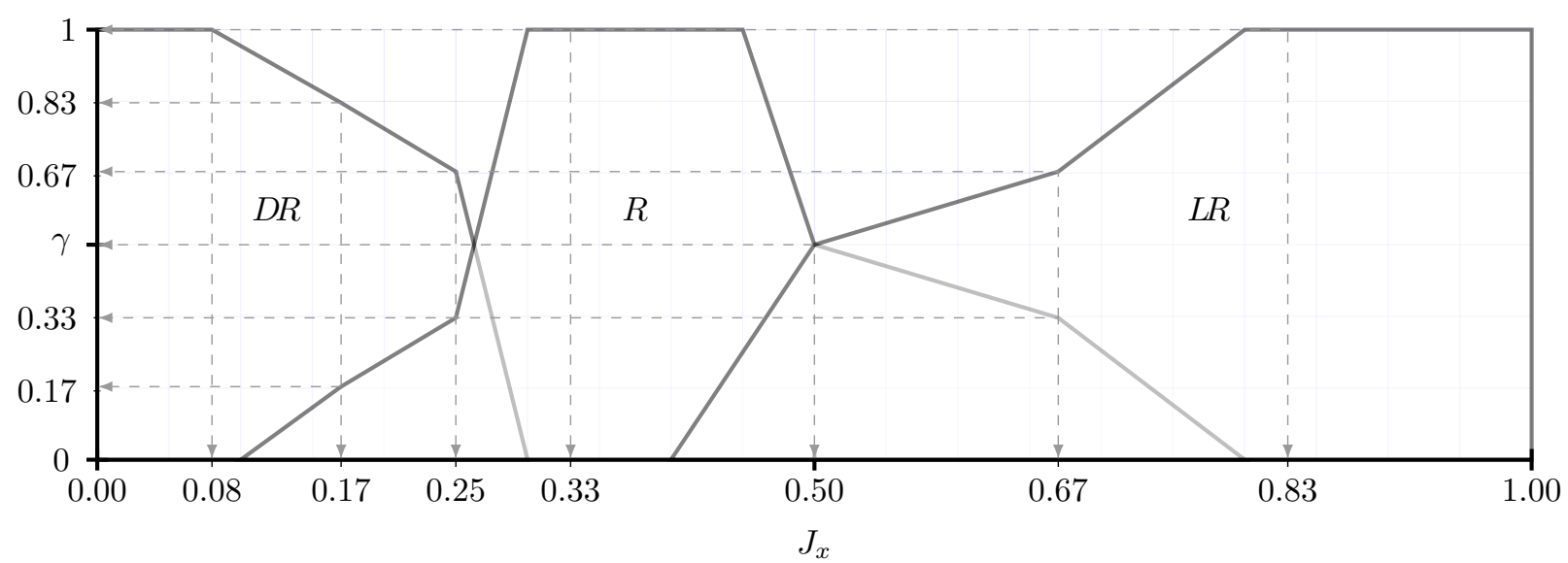

Figure 6: A possible continuous visualisation for Example 1, based on all the generated significance measures for the R-fuzzy sets of Dark Red, Red \& Light Red

Example 2: Assume that $F=\left\{f_{1}, f_{2}, \ldots, f_{9}\right\}$ is a set containing 9 different colour swatches based on the colour blue:
$f_{1} \rightarrow$
$[25,25,112] \rightarrow$
$f_{2} \rightarrow$
$[204,204,255] \rightarrow$
$f_{3} \rightarrow$
$[42,82,190] \rightarrow$
$f_{4} \rightarrow$
$[0,51,153] \rightarrow$
$f_{5} \rightarrow$
$[0,0,128] \rightarrow$
$f_{6} \rightarrow$
$[0,0,255] \rightarrow$
$f_{7} \rightarrow$
$[40,146,172] \rightarrow$
$f_{8} \rightarrow$
$[0,128,255] \rightarrow$
$f_{9} \rightarrow$
$[36,186,255] \rightarrow$

The example investigates the collected perception of 20 individuals, with regards to the colour blue. The more observations contained in the criteria set, the greater the chances of including disjoint distribution, where a particular R-fuzzy set is comprised of more than one area of distribution. The more individuals to give their perception, the more likely confliction may arise. These collected perceptions are presented in Table 2. Notice the inclusion of the Sex attribute, of which there are 15 males and 5 females.

In much the same way for that of Example 1, the $[\mathrm{RGB}]$ values are used, from which the averages are taken: $N=\{54,221,105,68,43,85,119,128,159\}$. These are processed using the same linear function as before, given by Eq. (19). The resulting fuzzy membership set is given as follows: 
Table 2: Human based perception for the variations of the colour blue

\begin{tabular}{|c|c|c|c|c|c|c|c|c|c|c|}
\hline \# & Sex & $f_{1}$ & $f_{2}$ & $f_{3}$ & $f_{4}$ & $f_{5}$ & $f_{6}$ & $f_{7}$ & $f_{8}$ & $f_{9}$ \\
\hline $\mathrm{p}_{1}$ & $M$ & $D B$ & $L B$ & $L B$ & $D B$ & $D B$ & $B$ & $L B$ & $L B$ & $B$ \\
\hline $\mathrm{p}_{2}$ & $F$ & $D B$ & $L B$ & $L B$ & $B$ & $D B$ & $B$ & $L B$ & $L B$ & $L B$ \\
\hline $\mathrm{p}_{3}$ & $M$ & $D B$ & $L B$ & $L B$ & $D B$ & $D B$ & $B$ & $L B$ & $L B$ & $L B$ \\
\hline $\mathrm{p}_{4}$ & $M$ & $D B$ & $L B$ & $B$ & $D B$ & $D B$ & $B$ & $L B$ & $L B$ & $L B$ \\
\hline $\mathrm{p}_{5}$ & $M$ & $D B$ & $L B$ & $B$ & $D B$ & $D B$ & $B$ & $L B$ & $L B$ & $L B$ \\
\hline $\mathrm{p}_{6}$ & $M$ & $D B$ & $L B$ & $B$ & $D B$ & $D B$ & $B$ & $D B$ & $L B$ & $L B$ \\
\hline $\mathrm{p}_{7}$ & $F$ & $D B$ & $L B$ & $B$ & $D B$ & $D B$ & $B$ & $L B$ & $B$ & $L B$ \\
\hline $\mathrm{p}_{8}$ & $F$ & $D B$ & $L B$ & $D B$ & $D B$ & $D B$ & $B$ & $D B$ & $L B$ & $L B$ \\
\hline $\mathrm{p}_{9}$ & $M$ & $D B$ & $L B$ & $B$ & $B$ & $D B$ & $B$ & $L B$ & $L B$ & $L B$ \\
\hline $\mathrm{p}_{10}$ & $M$ & $D B$ & $L B$ & $B$ & $D B$ & $D B$ & $B$ & $L B$ & $L B$ & $L B$ \\
\hline $\mathrm{p}_{11}$ & $M$ & $D B$ & $L B$ & $B$ & $D B$ & $D B$ & $B$ & $L B$ & $L B$ & $L B$ \\
\hline $\mathrm{p}_{12}$ & $M$ & $D B$ & $L B$ & $B$ & $B$ & $D B$ & $B$ & $L B$ & $B$ & $L B$ \\
\hline $\mathrm{p}_{13}$ & $M$ & $D B$ & $L B$ & $B$ & $B$ & $D B$ & $B$ & $L B$ & $B$ & $L B$ \\
\hline $\mathrm{p}_{14}$ & $M$ & $D B$ & $L B$ & $D B$ & $D B$ & $D B$ & $B$ & $L B$ & $L B$ & $L B$ \\
\hline $\mathrm{p}_{15}$ & $M$ & $D B$ & $L B$ & $D B$ & $D B$ & $D B$ & $B$ & $L B$ & $L B$ & $L B$ \\
\hline $\mathrm{p}_{16}$ & $F$ & $D B$ & $L B$ & $D B$ & $D B$ & $D B$ & $B$ & $L B$ & $B$ & $L B$ \\
\hline $\mathrm{p}_{17}$ & $F$ & $D B$ & $L B$ & $B$ & $B$ & $D B$ & $B$ & $L B$ & $L B$ & $L B$ \\
\hline $\mathrm{p}_{18}$ & $M$ & $D B$ & $L B$ & $D B$ & $D B$ & $D B$ & $B$ & $L B$ & $B$ & $L B$ \\
\hline $\mathrm{p}_{19}$ & $M$ & $D B$ & $L B$ & $D B$ & $D B$ & $D B$ & $B$ & $L B$ & $L B$ & $L B$ \\
\hline $\mathrm{p}_{20}$ & $M$ & $D B$ & $L B$ & $B$ & $B$ & $D B$ & $B$ & $L B$ & $L B$ & $L B$ \\
\hline
\end{tabular}

$$
J_{x}=\{0.06,1.00,0.35,0.14,0.00,0.24,0.43,0.48,0.65\}
$$

The descriptor terms contained within the table have the following associated meaning:

$$
D B \rightarrow \text { Dark Blue } \quad B \rightarrow \text { Blue } \quad L B \rightarrow \text { Light Blue }
$$


Using Definition 6, the final generated R-fuzzy sets based on the collected subsets for $L B, B$ and $D B$, respectively, are given as:

$$
\begin{aligned}
D B & =(\{0.00,0.06\},\{0.00,0.06,0.14,0.35,0.43\}) \\
B & =(\{0.24\},\{0.14,0.24,0.35,0.48,0.65\}) \\
L B & =(\{1.00\},\{0.35,0.43,0.48,0.65,1.00\})
\end{aligned}
$$

Making use of the significance measure given by Eq. (12), allows for one to ascertain the significance of each and every encapsulated fuzzy membership value, for each R-fuzzy set, the results of which are presented

\begin{tabular}{|c|c|c|c|c|c|}
\hline$D B$ & & $B$ & & $L B$ & \\
\hline$J_{x}$ & $\gamma$ & $J_{x}$ & $\gamma$ & $J_{x}$ & $\gamma$ \\
\hline$\gamma \overline{D B}\{0.00\}=$ & 1.00 & $\gamma \bar{B}\{0.00\}=$ & 0.00 & $\gamma \overline{L B}\{0.00\}=$ & 0.00 \\
\hline$\gamma \overline{D B}\{0.06\}=$ & 1.00 & $\gamma \bar{B}_{B}\{0.06\}=$ & 0.00 & $\gamma \overline{L B}\{0.06\}=$ & 0.00 \\
\hline$\gamma \overline{D B}\{0.14\}=$ & 0.70 & $\gamma \bar{B}_{B}\{0.14\}=$ & 0.30 & $\gamma \overline{L B}\{0.14\}=$ & 0.00 \\
\hline$\gamma \overline{D B}\{0.24\}=$ & 0.00 & $\gamma \bar{B}\{0.24\}=$ & 1.00 & $\gamma \overline{L B}\{0.24\}=$ & 0.00 \\
\hline$\gamma \overline{D B}\{0.35\}=$ & 0.30 & $\gamma \bar{B}_{B}\{0.35\}=$ & 0.55 & $\gamma \overline{L B}\{0.35\}=$ & 0.15 \\
\hline$\gamma \overline{D B}\{0.43\}=$ & 0.10 & $\gamma \bar{B}_{B}\{0.43\}=$ & 0.00 & $\gamma \overline{L R}\{0.43\}=$ & 0.90 \\
\hline$\gamma \overline{D B}\{0.48\}=$ & 0.00 & $\gamma \bar{B}_{B}\{0.48\}=$ & 0.25 & $\gamma \overline{L B}\{0.48\}=$ & 0.75 \\
\hline$\gamma \overline{D B}\{0.65\}=$ & 0.00 & $\gamma_{\bar{B}}\{0.65\}=$ & 0.05 & $\gamma \overline{L B}\{0.65\}=$ & 0.95 \\
\hline$\gamma \overline{D B}\{1.00\}=$ & 0.00 & $\gamma \bar{B}\{1.00\}=$ & 0.00 & $\gamma \overline{L B}\{1.00\}=$ & 1.00 \\
\hline
\end{tabular}
in Table 3.

Table 3: The degrees of significance based on Table 2

Figure 7 provides one with a derivable continuous representation of the generated R-fuzzy sets and the returned degrees of significance. Given that $\gamma \overline{D B}\{0.24\}=0.00$, there exists an area of disjointness between 


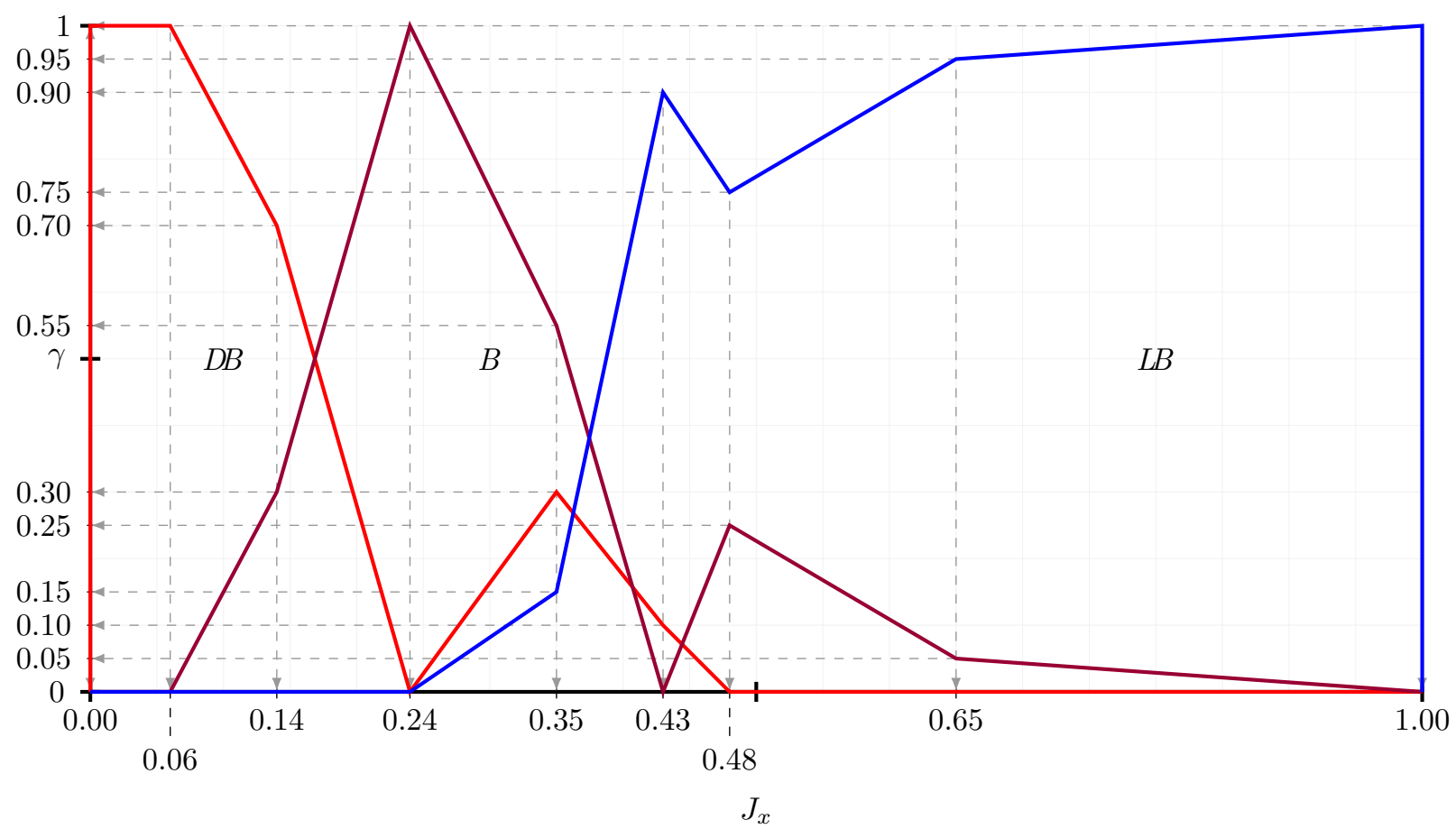

Figure 7: A derivable continuous visualisation for Example 2, based on all the generated significance measures for the R-fuzzy sets of $D B, V \& L B$

0.14 and 0.35. Referring back to Table 3, one can clearly see where these areas of disjointness occur and how they are reflected in Figure 7. The R-fuzzy set $L B$ has considerable variance throughout its duration, as can be seen in its fluctuations, it does not however have an area of disjointness. All three generated R-fuzzy sets do have at least one value which returned a significance degree of 1 , so even with the extra members for the criteria set, there is still a value that exists indicative of the collective perception held.

As the fuzzy membership set $J_{x}$ remains constant throughout, by that, the same membership is used for all generated R-fuzzy sets, the sequence curve of each R-fuzzy set can be made use of when considering the absolute degree of grey incidence. The fuzzy membership values themselves act as the discretised anchor points along the $x$-axis. The amplitude of each significance measure score registers on the $y$-axis. Therefore, one is able to present a two dimensional sequence, given over a universe of discourse that will remain the same for all generated R-fuzzy sets and subsets. If a sequence from one R-fuzzy set is compared to that of another, one will be able to provide a metric value to the divergence and similarities that exists between comparable sequences. Whether the differences are small or large is irrelevant, but rather there is a difference to begin with, this should warrant further investigation and inference.

As the data contained in Table 2 contains a Sex attribute, one can generate two subsets with relation to male and female. This allows for one to create R-fuzzy subsets, from which the returned degrees of 
Table 4: The Degrees of Significance for Males

\begin{tabular}{|c|c|c|c|c|c|}
\hline$D B$ & & $B$ & & $L B$ & \\
\hline$J_{x}$ & $\gamma$ & $J_{x}$ & $\gamma$ & $J_{x}$ & $\gamma$ \\
\hline$\gamma \overline{D B}\{0.00\}=$ & 1.00 & $\gamma_{\bar{B}}\{0.00\}=$ & 0.00 & $\gamma \overline{L B}\{0.00\}=$ & 0.00 \\
\hline$\gamma \overline{D B}\{0.06\}=$ & 1.00 & $\gamma_{\bar{B}}\{0.06\}=$ & 0.00 & $\gamma \overline{L B}\{0.06\}=$ & 0.00 \\
\hline$\gamma \overline{D B}\{0.14\}=$ & 0.73 & $\gamma_{\bar{B}}\{0.14\}=$ & 0.27 & $\gamma \overline{L B}\{0.14\}=$ & 0.00 \\
\hline$\gamma \overline{D B}\{0.24\}=$ & 0.00 & $\gamma_{\bar{B}}\{0.24\}=$ & 1.00 & $\gamma \overline{L B}\{0.24\}=$ & 0.00 \\
\hline$\gamma \overline{D B}\{0.35\}=$ & 0.27 & $\gamma_{\bar{B}}\{0.35\}=$ & 0.60 & $\gamma \overline{L B}\{0.35\}=$ & 0.13 \\
\hline$\gamma \overline{D B}\{0.43\}=$ & 0.07 & $\gamma_{\bar{B}}\{0.43\}=$ & 0.00 & $\gamma_{\overline{L R}}\{0.43\}=$ & 0.93 \\
\hline$\gamma \overline{D B}\{0.48\}=$ & 0.00 & $\gamma_{\bar{B}}\{0.48\}=$ & 0.20 & $\gamma \overline{L B}\{0.48\}=$ & 0.80 \\
\hline$\gamma \frac{}{D B}\{0.65\}=$ & 0.00 & $\gamma_{\bar{B}}\{0.65\}=$ & 0.07 & $\gamma \overline{L B}\{0.65\}=$ & 0.93 \\
\hline$\gamma \overline{D B}\{1.00\}=$ & 0.00 & $\gamma_{\bar{B}}\{1.00\}=$ & 0.00 & $\gamma \overline{L B}\{1.00\}=$ & 1.00 \\
\hline
\end{tabular}

significance can be used to generate the comparable sequences needed, so that the absolute degree of grey incidence can be deployed. Regardless of how the encapsulation of the returned degrees of significance look, it still provides a valid sequence for comparisons to be undertaken, as any generated subset will be indicative of the overall R-fuzzy sets generated from it, along with disjointness and all.

Table 4 and Table 5, contain the returned degrees of significance for males and females, respectively, collected from the perceptions recorded in Table 2. The plots contained in Figure 8, Figure 9 and Figure 10, show the comparable sequences computed from the returned degrees of significance, for $D B, B$ and $L B$. Table 6 provides one with a digestive summary of the collected absolute degree of grey incidence values for all generated R-fuzzy sets and significance measure sequences, for each comparable permutation, of which there are 3. Inspecting the table one can see that sequences generated for $D B$ shared the most similarities with a returned metric of $\epsilon(0.968)$. This was then followed by $L B$, with a metric of $\epsilon(0.940)$, therefore the greatest divergence exists for $B$, with a metric of $\epsilon(0.841)$. 


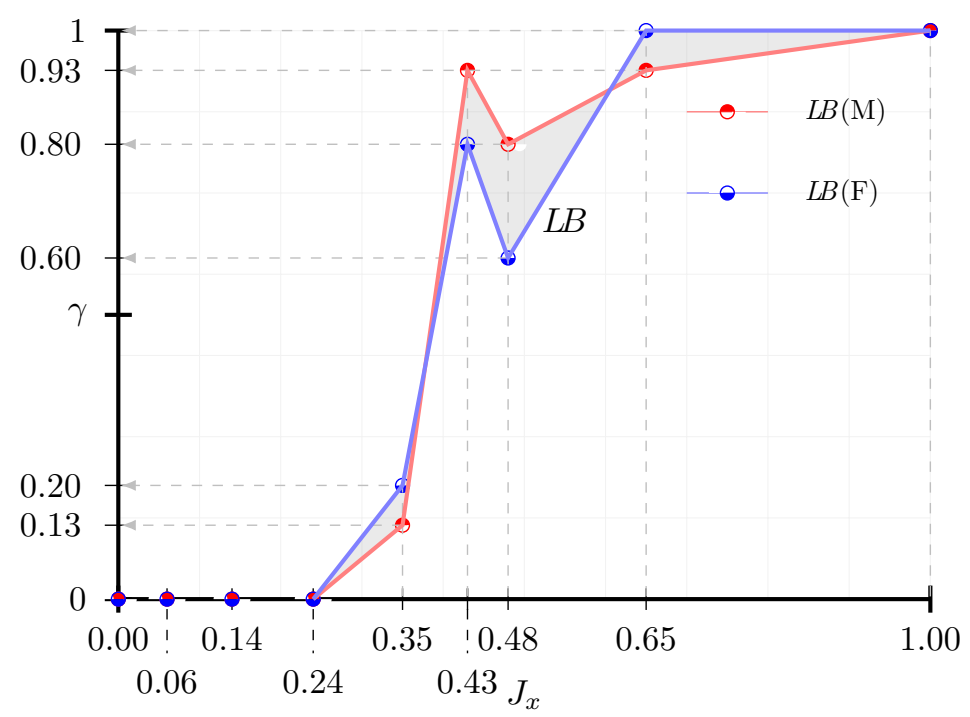

Figure 8: The Comparability Between Males and Females for $L B$

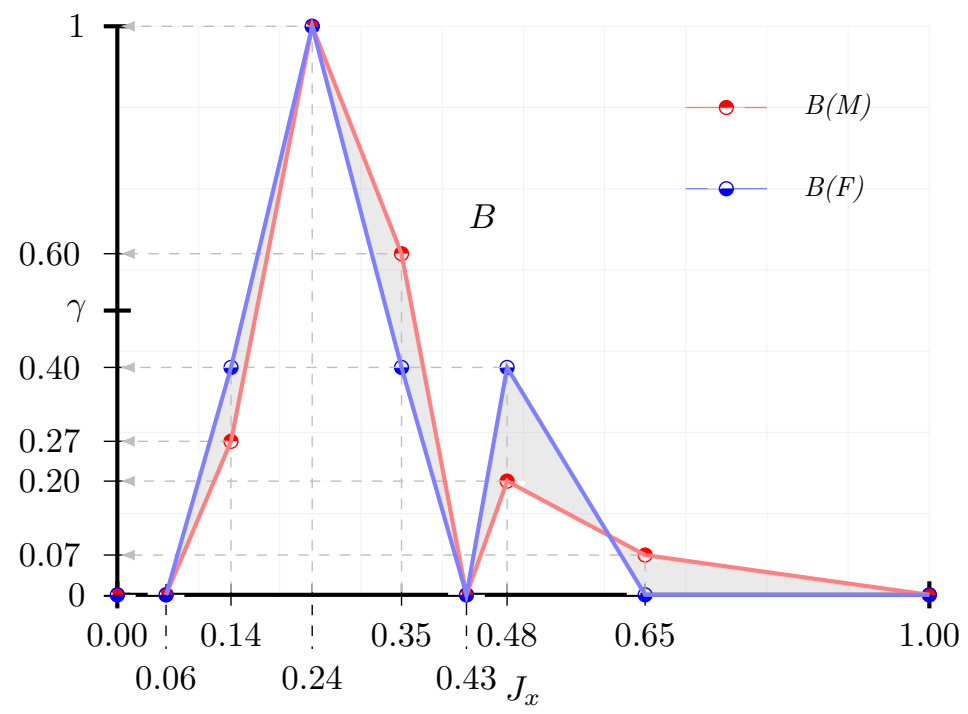

Figure 9: The Comparability Between Males and Females for $B$

Based on the experimental outcomes of the presented examples, we would like to emphasise how such a framework is able to provide for a wealth of higher detailed information. Such results can effectively be used as a template to possibly use to forecast how a collective, or demographic of people may perceive an anticipated observation. If the population was to contain a variety of different age classifications, and given the same initial observation, one could possibly identify a rate of change between age groups. If that was to hold true, one could then use that information to better cater for expectations. This could be given more credence when considering additional attributes, for example; sex, nationality, ethnicity and so on. Any 
Table 5: The Degrees of Significance for Females

\begin{tabular}{|c|c|c|c|c|c|}
\hline$D B$ & & $B$ & & $L B$ & \\
\hline$J_{x}$ & $\gamma$ & $J_{x}$ & $\gamma$ & $J_{x}$ & $\gamma$ \\
\hline$\gamma \overline{D B}\{0.00\}=$ & 1.00 & $\gamma_{\bar{B}}\{0.00\}=$ & 0.00 & $\gamma \overline{L B}\{0.00\}=$ & 0.00 \\
\hline$\gamma \overline{D B}\{0.06\}=$ & 1.00 & $\gamma \bar{B}\{0.06\}=$ & 0.00 & 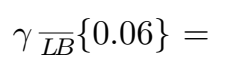 & 0.00 \\
\hline$\gamma \overline{D B}\{0.14\}=$ & 0.60 & $\gamma_{\bar{B}}\{0.14\}=$ & 0.40 & $\gamma \frac{}{L B}\{0.14\}=$ & 0.00 \\
\hline$\gamma \overline{D B}\{0.24\}=$ & 0.00 & $\gamma_{\bar{B}}\{0.24\}=$ & 1.00 & $\gamma \overline{L B}\{0.24\}=$ & 0.00 \\
\hline$\gamma \frac{}{D B}\{0.35\}=$ & 0.40 & $\gamma_{\bar{B}}\{0.35\}=$ & 0.40 & $\gamma \overline{L B}\{0.35\}=$ & 0.20 \\
\hline$\gamma \overline{D B}\{0.43\}=$ & 0.20 & $\gamma_{\bar{B}}\{0.43\}=$ & 0.00 & $\gamma \frac{}{L R}\{0.43\}=$ & 0.80 \\
\hline$\gamma \overline{D B}\{0.48\}=$ & 0.00 & $\gamma_{\bar{B}}\{0.48\}=$ & 0.40 & 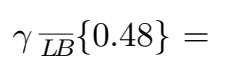 & 0.60 \\
\hline$\gamma \overline{D B}\{0.65\}=$ & 0.00 & $\gamma_{\bar{B}}\{0.65\}=$ & 0.00 & $\gamma_{\overline{L B}}\{0.65\}=$ & 1.00 \\
\hline$\gamma \overline{D B}\{1.00\}=$ & 0.00 & $\gamma_{\bar{B}}\{1.00\}=$ & 0.00 & $\gamma_{\overline{L B}}\{1.00\}=$ & 1.00 \\
\hline
\end{tabular}

Table 6: A Comparable Summary of the Returned Absolute Degree of Grey Incidence for $L B, B \& D B$

\begin{tabular}{c|cc|c|cc|c|cc}
\hline$L B$ & $\mathrm{M}$ & $\mathrm{F}$ & $B$ & $\mathrm{M}$ & $\mathrm{F}$ & $D B$ & $\mathrm{M}$ & $\mathrm{F}$ \\
\hline \hline $\mathrm{M}$ & $\epsilon(1.00)$ & $\epsilon(0.940)$ & $\mathrm{M}$ & $\epsilon(1.00)$ & $\epsilon(0.841)$ & $\mathrm{M}$ & $\epsilon(1.00)$ & $\epsilon(0.968)$ \\
$\mathrm{F}$ & - & $\epsilon(1.00)$ & $\mathrm{F}$ & - & $\epsilon(1.00)$ & $\mathrm{F}$ & - & $\epsilon(1.00)$
\end{tabular}

permutation of collected information based on the attributes could be used to model the initial population, from which sub-cohorts could be identified using any possible number of permutations. As the framework is based on the collecting of human perception, assuming that the populous provided truthful responses, for those individuals where the perceptions recorded are contradictory to the norm, this may indicate possible underlying conditions. Consider the colour based examples presented, in each case there was a single fuzzy 


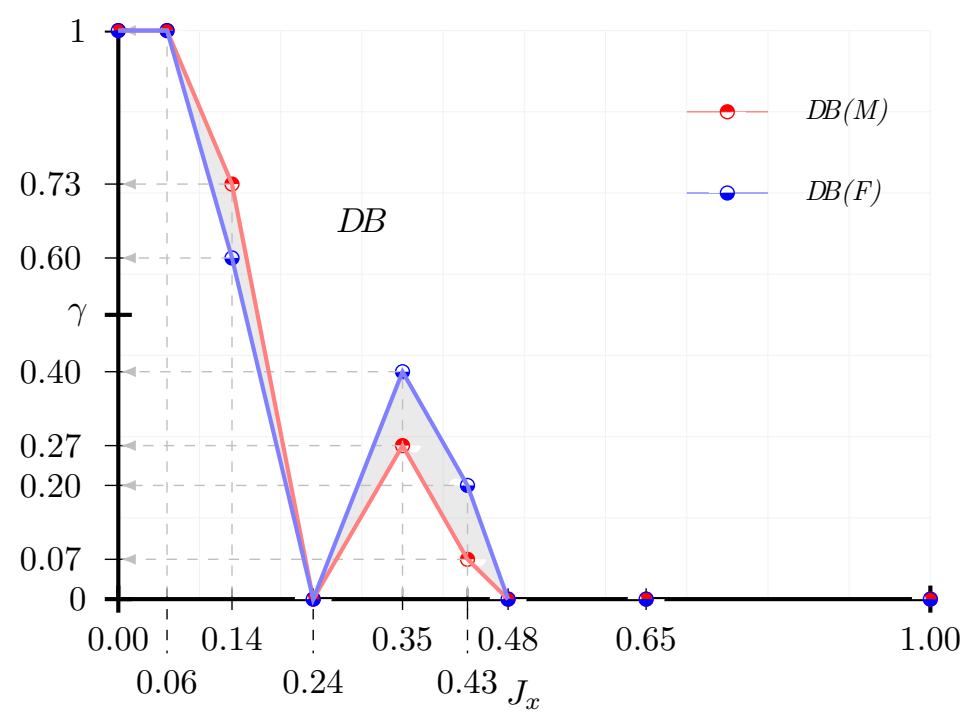

Figure 10: The Comparability Between Males and Females for $D B$

value that could be used to represent a particular R-fuzzy set. The distribution from that initial fuzzy value would reduce in significance, however, if a member of the populous generally felt that a particular membership value is indeed indicative of the R-fuzzy set being modelled, they may have different levels of perception completely, alluding to possible colour blindness. If it can be concluded that any member of the population responded in favour of a particular fuzzy membership value for a specifically created swatch or audio sample, then one would be able to identify individuals with inherent underlying conditions akin to colour blindness and audio deafness.

\subsection{Strengths and weaknesses}

There are indeed several other approaches one could adopt when considering modelling subjective based uncertainty, and there indeed strengths and weaknesses one should consider when deciding upon a chosen model. If you consider that a rough set from rough set theory is more associated to the notion of ambiguity and the lack of information, whereas a fuzzy set from fuzzy theory is more associated with vagueness and non-sharp definable boundaries, one will appreciate that the hybridising of these two concepts will provide for a more versatile model. There have been several models proposed that combines the benefits of rough and fuzzy methodologies; Bodjanova (2007), Deng et al. (2007), Dubois (1980), Dubois and Prade (1990), 45 Huynh and Nakamori (2005), Jensen and Shen (2008, 2009), Nanda and Majumdar (1992), Pawlak and Skowron (2007), Radzikowska and Kerre (2002), Sun et al. (2014), Wu et al. (2003), Xu et al. (2012), Zeng et al. (2015). However, these models mainly make use of similarity and equivalence relations, whereas the R-fuzzy set was the first of which to use roughs sets to approximate the membership of a fuzzy set.

Fuzzy is not the only consideration that one could apply in the context of providing a mathematical 
tool for subjective uncertainty quantification, there are several alternatives. One could consider the theory of evidence presented in Shafer (1976), based on epistemic probability and also possibility and imprecise probability theories. The proposed framework in this paper is associated to conditional probability, rather than exclusively epistemic probability, but probability nonetheless. The notion of weights of evidence is not too dissimilar in sentiment to the significance measure that links the R-fuzzy set to that of grey analysis, for without the significance measure the framework would simply not exist. The R-fuzzy and grey analysis framework is fuzzy based in its construction, therefore it inherits the degrees of membership to an extent rather than probability, invoking more plausibility and possibility. It is only when the significance measure is deployed does it act in a conditional probability sense. It is the collection of these instances that create the sequence that the grey component of the framework utilises to provide for greater analysis of the population that the framework was acted upon. There also exists hybrid uncertainty propagation as presented in Wang and Matthies (2019), this again makes use of epistemic uncertainty and evidence parameters with the output response being interpreted by an interval-type mean value with fuzzy bounds. The R-fuzzy framework instead employs a crisp rough bounding of uncertain fuzzy memberships values, from which the significance measure is then able to quantify the conditional probability of each captured value belonging to the membership set. Unlike the interval approach, no loss of information is incurred as it would when entering an interval. Using a rough bounding for a fuzzy membership value is stark contrast to typically using a fuzzy bounding on a crisp value. Another interval based approach is that of Muhanna and Mullen (2001), where the uncertainties are introduced as bounded possible intervals, an interval finite-element method. In much the same way as the previous interval method, the R-fuzzy framework approach does away with conventional intervals and instead incorporates a membership set comprised of a rough set whereby every contained value is crisp and uniquely identifiable. The most striking difference to all the approaches identified is the use of a set for the membership set, where every value contained in that set can be inspected and the conditional probability calculated. When compared to the use of intervals, one loses some resolution with regards to uniqueness, for any value contained within an interval will lose its discernibility.

It can be seen from the literature that a predominately fuzzy based approach does pay dividends when exploring uncertainty in data; (Amirkhan et al., 2018), Salmeron (2010), Melin et al. (2018), Tiwari et al. (2018), de S. Sampaio Filho et al. (2018), Dwivedi et al. (2018). The literature is rife with fuzzy based approaches and this paper maintains this fuzzy perspective.

The R-fuzzy pairing with the significance measure provided for an alternative approach for garnering higher levels of detail that would not otherwise be possible when considering existing approaches. If a type-2 fuzzy problem can be translated to an R-fuzzy problem, then the addition of the significance measure would allow for an intermediary approach to that of a generalised type-2 fuzzy set. It is generally agreed upon that a generalised type-2 approach is the most apt at capturing higher levels of detail, but due to its unfavourable computational burden, the interval type-2 fuzzy approach is instead adopted (Mendel and John, 2002). 
With the inclusion of grey analysis, which completes the RfGAf, the level of post-analysis that can be undertaken provides for additional levels of detailed analysis. Without the significance measure, one would not be able to connect R-fuzzy sets to that of grey analysis.

The grey analysis component was specifically chosen for its ability to work on data sequences of small magnitudes. In addition, the authors have considerable experience with grey theory, and its applicability is not as widely known as one would like, ergo, the introduction of grey theory into the framework. The absolute degree of grey incidence is the actual grey attribute from the paradigm that is used, it is this component that is key to the entirety of the RfGAf. As it will be seen, the additional post-analysis of the results using a grey approach can provide for improved inferencing.

The complete framework mimics a generalised type-2 fuzzy approach with just using an R-fuzzy set and significance measure pairing, but extends on this to provide even more resolution when introducing the grey analysis component. For it is the grey incidence analysis that allows for one to compare and contrast any permutation of contained conditional attributes present in the data.

If it can be shown that the RfGAf is a viable candidate for uncertainty modelling, specifically with regards to subjective perception based uncertainty, then the applicability of R-fuzzy sets and the RfGAf will gain a stronger footing. This paper has intrinsically described and demonstrated the effectiveness of the proposed framework, via the use of examples and scenarios.

The main strength lies in the framework's ability to capture and maintain the subjectiveness of the population. The robustness and scalability provides another advantage, the framework can be easily deployed on a population as small as a single individual, to that of an extremely large cohort. If the population contains little to no variance, this will likely result in the comparable sequences being very similar to, or identical, resulting in a 1 , or very close to a 1 , for its absolute degree of grey incidence. If however that population contains a great deal of variance, then one can expect to see more divergence in the returned absolute degree of grey incidence. The level of resolution when compared to the aforementioned approaches is greater when considering the RfGAf, as the crisp uncertain fuzzy values belonging to the membership set of each and every R-fuzzy set are what the conditional probabilities are derived for. Therefore, each member of the set can be uniquely identified and extrapolated, and compared and contrasted against other contained sub-R-fuzzy sets from the same population. As it has been seen from the showcased examples, a wealth of information can still be garnered from such a small consignment of data.

As with any proposed framework there are indeed weaknesses. The RfGA framework utilises an arbitrary number of R-fuzzy sets, if one was to make use of too few R-fuzzy sets then one would not garner any information of real significance. Equally, if one was to implement too many R-fuzzy sets, then the collected results could possibly contain too much divergence, however, the divergence and convergence could still be quantified. More consideration needs to be given when deciding on the number of R-fuzzy sets to make use of. As with any model regarding subjective based uncertainty, the responses of the population could change 
unexpectedly. If one was to present a question concerning an observation and the responses were recorded, and the same observation was presented a short while later, the responses given may not be the same as the initial responses. However, this is completely acceptable, to try and force and illicit a response skews and falsifies the results. Human nature and response is instinctive and may not necessarily be repetitive. The other approaches are significantly more well-known and therefore have had greater applicability in a variety of different domains. The proposed framework lacks any significant application area due to its infancy, however the authors will continue to improve upon the global applicability of the framework through future research and applications.

\section{Relationships with fuzzy}

In this section, the relationships to that of the fuzzy in general are discussed. In particular, it is the

Theorem 1. The significance measure as presented in Definition 7 is equivalent to that of a type-1 fuzzy set as defined in Definition 1, only if it can be described in much the same way. Whereby an object belonging to its membership set can be described by its returned value, such that it is either in the ranger of or equal to $[0,1]$. In addition, for the equivalence to be maintained, the continuous set representation of the significance notion of the R-fuzzy and significance measure pairing that is described, as it is this concept which has the greatest association to traditional fuzzy means.

It should be understood that R-fuzzy sets are inherently different from traditional fuzzy sets. An R-fuzzy set will express its membership values as a set, akin to a type-2 fuzzy set, whereas a type-2 fuzzy set will express its membership value as a singleton value. There are also indeed similarities between fuzzy and that of the R-fuzzy and significance pairing. In their original work, Yang and Hinde (2010) presented the proofs and theorems for the relationships that were found to that of R-fuzzy and type-1 fuzzy sets; intervalvalued fuzzy sets; and Atanassov intuitionistic fuzzy sets. We will begin with describing the relation of that of the significance measure as presented in Definition 7 , to that of a type- 1 fuzzy set as described in Definition 1. We will also describe the relationship of an R-fuzzy set and significance measure pairing to that of a generalised type-2 fuzzy, as defined in Definition 2.

In Yang and Hinde (2010), it was clearly stated that if attempts to quantify the distribution of the membership function of the R-fuzzy were successful, then one would then be able to derive a type-1 fuzzy set, which itself would then allow for one to cater for a link to a type-2 fuzzy set. As one can easily infer from the significance measure presented in Definition 7, it does indeed act as an equivalent type-1 fuzzy set, in that it provides an object value a degree of inclusion to the R-fuzzy descriptor.

measure, must always be structured on the amplitude of the stick heights of the returned degrees of significance for all encapsulated fuzzy membership values. 
Proof 1. From the degree of significance in Definition 7 and the R-fuzzy set as presented in Definition 6 , assume that $A$ is an R-fuzzy set descriptor describing a particular notion of subjectivity. A type-1 fuzzy set is a set of ordered pairs, providing the object value with an accompanying degree of membership. The same restrictions that apply to a type-1 fuzzy set also apply to the objects of an $R$-fuzzy set, in that they cannot exceed and can only be contained within [0,1]. In much the same way an object's membership is described by a fuzzy membership function, the degree of significance for all associated $R$-fuzzy set membership values is given by its membership function; $\gamma_{\bar{A}}\{v\}: J_{x} \rightarrow[0,1]$, given by the following expression:

$$
\bar{A}=\left\{\left\langle v, \gamma_{\bar{A}}\{v\}\right\rangle \mid v \in J_{x}\right\}
$$

Given the expression, one can see that the membership set will constitute pairs of objects and their accompanying degrees of significance, or simply, the conditional probability of an object in relation to the criteria set and the descriptor. This expression is an equivalence to the formal expression presented in Eq. (1):

$$
A=\left\{\left\langle x, \mu_{A}(x)\right\rangle \mid x \in \mathbb{U}\right\}
$$

Where an object is presented with a degree of association in relation to the set that it is being modelled for. Inspecting $v \in J_{x}$, which relates the membership set of fuzzy membership values, in much the same way that $x \in \mathbb{U}$, is representative of the objects belonging to the universe of discourse. In the case of $J_{x}$, this is the equivalent universe of discourse as membership values belonging $v \in J_{x}$ will always constitute the discretised points of the $x$-axis. It can be seen that the significance measure is an equivalence to that of a type-1 fuzzy set, only when the set is the representative of the $R$-fuzzy descriptor that the $R$-fuzzy set was being modelled for.

A type-2 fuzzy set as described in Definition 2 can be seen as a logical extension to that of a type-1 fuzzy set, by including this notion of a secondary grade of membership. The additional attribute itself is that of a type-1 fuzzy membership, in doing so, a 3-dimensional representation can now be achieved.

$$
\tilde{A}=\left\{\left\langle(x, u), \mu_{\tilde{A}}(x, u)\right\rangle \mid \forall x \in \mathbb{U}, \forall u \in J_{x} \subseteq[0,1]\right\}
$$

Theorem 2. An R-fuzzy set $A$ is equivalent to a type-2 fuzzy set as described in Definition 2, only if the notion of Theorem 1 is considered, such that the conditional probability of the degree of significance is equivalent to that of a type-1fuzzy set. Therefore, it can be seen that an R-fuzzy set does indeed have an equivalence to a type-2 fuzzy set with a discrete secondary grade of membership.

Proof 2. From the type-2 fuzzy set as presented in Definition 2, $(x, u)$ is representative of an intersection and $\mu_{\tilde{A}}(x, u)$ is representative of the amplitude of an object's association to the secondary grade of membership for that particular intersection. From the definition of an R-fuzzy set as described in Definition 6 , 
one can see that a rough set it utilised as the membership set of an R-fuzzy set, comprised of the lower and upper approximations $\left(\underline{M}_{A}, \bar{M}_{A}\right)$, respectively. The degree of significance as described in Definition 7 and given in Eq. (15). Where the calculated degree of significance is given by $\gamma_{\bar{A}}\{x\}$, for each and every included object belonging to the membership set of the R-fuzzy set it is associated to. In the same way that $\mu_{\tilde{A}}(x, u)$ from a type-2 fuzzy set provides one with the amplitude of the object with regards to its secondary grade of membership, $\bar{A}$ provides one with the collected degrees of significance for all uncertain fuzzy membership values that were encapsulated in relation to the R-fuzzy set they were associated to. Both approaches utilise a set as a membership set, where the distribution of said sets is described.

\section{Remarks}

As one can see from Theorem 2 and Proof 2, the strengths of the significance measure when hybridised with that of an R-fuzzy set, provides one with a bridge to that of a generalised type-2 fuzzy approach. The most common irritation one would associate to a generalised type- 2 approach is that of the exponential computational burden one becomes aware of when incorporating additional dimensions of uncertainty encapsulation. The complexity increases when trying to capture more uncertainty. A common trait of uncertain systems is that one may not necessarily know all with regards to the problem domain, so securing a precise foundation from which to infer from becomes unrealistic. The qualities of an R-fuzzy set allows for one to capture subjective uncertainty, both individualised nuances and a generalised consensus, when exactness of the problem is not there. The addition of the significance measure to then quantify the distribution of captured uncertain fuzzy membership values for any given R-fuzzy set, provides one with the ability to capture additional subjectivity in the amplitude of the membership values. Therefore the quality of a captured value can be inferred from to provide a more detailed response. As the significance measure acts as a type-1 fuzzy set, R-fuzzy sets provide a bridge connecting an equivalence in representation which would otherwise need a type- 2 membership in a type-2 fuzzy set. It can be agreed upon that memberships of a type-1 nature are more indicative of objectiveness, whereas type-2 memberships will be more indicative of a subjective nature. What is lacking is a reliable methodology to set up such a type-2 membership. This paper has presented one with a practical means to provide for an effective type-2 representation with a significantly reduced computational burden. Given this perspective, the R-fuzzy and significance component of the proposed framework caters for an intermediary approach, to allow for greater swathes of subjective uncertainty to be encapsulated and inferred from.

As can be seen from Example 1 and Example 2, human based perception is inherently subjective, and as a result may not follow a general rule of thumb. Individual subjectivity is based on the individual and not necessarily the consensus of the group. Such is the versatility of an R-fuzzy set, one is able to capture uncertainty associated to an individual and to also capture the uncertainty of the group, all within a single 
R-fuzzy set, for any number of descriptors.

The proposed framework is not without its own drawbacks, it is with regards to the criteria set $C$ and the fuzzy membership set $J_{x}$. If any changes are made to either of these two sets, the results of the R-fuzzy sets based on them will also change. As the proposed new framework is very algorithmic, the order in which the processes are undertaken are crucial, as with all other frameworks. Depending on the volatility of the domain, some areas of application will be more preferable than others.

It is the problem of making use of excessive preciseness to describe ever increasing imprecise phenomena, that has seen the proposal of several new approaches to capture higher degrees of uncertainty, approaches and notions already mentioned. Such as; Atanassov intuitionistic fuzzy sets, interval-valued fuzzy sets, type-2 fuzzy sets and shadowed sets. The relationship to these approaches have been described by Yang and Hinde (2010), and the relationship to type-2 fuzzy sets has been presented in this paper. The main pitfall from these approaches is that they assume uniformed distribution of collected elements. This is absolutely not the case as the examples in this paper have shown. Subjectivity cannot be treated with strictness and uniformity, doing so would certainly skew the results.

The differences and similarities one can have for the same given observation, should all be taken into consideration. It is clear to see from Example 1 and Example 2, such differences and similarities are prevalent even in a relatively small criteria set. Given that a single crisp value derived from a type-1 fuzzy set, using such a value to be indicative of a descriptive notion, would not be ideal as it would be somewhat skewed, as it was demonstrated when using the average. Given that the membership of an R-fuzzy set is a set itself, which constitutes discrete fuzzy membership values, there is no loss of information at any stage. When you consider intervals, any object to fall within it bounded region will lose all uniqueness, there is no indiscernibility between the lower and upper bounds of the interval itself. As the membership values that make up the lower and upper approximation of an R-fuzzy set are crisp, one is then able to quantify the distribution and the significance of each collected value; ergo the significance measure.

What was remarked upon in the original paper that proposed the significance measure was the notion of an $\alpha$-plane to aid in the post analysis, by providing horizontal slices at all levels of significance, but with particular interest to those areas associated to the extremes. Given that the number of observers in the criteria set $C$ for any generated R-fuzzy set will be of the same magnitude, this enforces that the same increments of significance are maintained. This would stay the same for any number of R-fuzzy sets generated from this criteria set. Consider Example 1 where there are 6 individuals contained in the criteria set $C$, therefore the only possible returned degrees of significance are as follows: $\{0,0.17,0.33,0.5,0.67,0.83,1\}$ $(0 / 6,1 / 6,2 / 6, \ldots, 6 / 6)$. Given that the extreme values in any case will always be that of a significance of either a 0 or 1 , one should then consider the degrees of significance directly above and below. Therefore, consider the degree of significance of 0.83 , which is $5 / 6$. If 5 out of 6 people agreed to the membership value in question, then one may be inclined to promote this value to the lower approximation and upgrade 
its returned degree of significance to a 1 which would signify absolute inclusion. Consider 0.17 , which is $1 / 6$, if only a single individual agreed to the membership value as an indicative representation, one may be inclined to demote this to a 0 and remove the membership from the upper approximation and the R-fuzzy set completely which would signify absolute non-inclusion. A more detailed explanation of the $\alpha$-plane notion can be found in the original doctoral thesis that proposed the significance measure (Khuman, 2016).

The R-fuzzy and grey analysis framework, as described in this paper has been shown to encapsulate a greater amount of subjective uncertainty than current approaches. It has also be shown that the framework can act as an intermediary approach to that of a full generalised type-2 fuzzy set. The reduction in complexity of computational processes, we believe, makes our proposed framework more superior in modelling subjective perception based domains.

\section{Conclusion}

This paper has put forward a new framework for encapsulating and inferencing from subjective based uncertainty - the R-fuzzy grey analysis framework (RfGAf). The constitutional parts of the framework are made up of; an R-fuzzy set foundation, to capture the subjective nature of human based perception for any given observation; the significance measure, to provide the quantification of the encapsulated uncertain fuzzy membership values and measure their distribution; grey analysis, to provide one with additional postanalysis of combinatorial permutations of nested R-fuzzy subsets. The sheer amount of uncertainty that a stand alone R-fuzzy set can capture, puts it in a favourable position to be expanded upon. This was the main attribute that encouraged further exploration of what an R-fuzzy set could be capable of. The introduction of the significance measure by (Khuman et al., 2015a) (Khuman, 2016), provided the means to quantify the distribution of the encapsulated fuzzy membership values and in doing so, created an intermediary approach to that of a generalised type-2 fuzzy set, with a significantly reduced computational burden. The introduction of the absolute degree of grey incidence from grey analysis, enabled for post-analysis to be undertaken, allowing for more detailed inferencing of the results. If the criteria set $C$ contains characteristic attributes, these can be used to generate R-fuzzy subsets, where the metric spaces between their geometric curves can be quantified to provide one with a degree of incidence, indicative of how similar or dissimilar comparable subsets are.

Considering the examples contained in this paper, one can see that a wealth of knowledge can still be garnered from a relatively small data set. A single observation can have varying perception associated to it; what is acceptable; e to some may not be acceptable for others. As is the nature of human based subjectivity, one cannot enforce their own subjectiveness onto others, the response should always be taken into consideration no matter how outlandish or against the grain of the common held norm it may be. Such responses may even be indicative of underlying issues. Consider the colour swatch examples presented in 
this paper, if the criteria set $C$ contained individuals whom suffered from a variation of colour blindness, their returned responses may be tell-tale signs of that particular variation. This could be further explored and is one of the research directions that the authors would like to consider for future work.

One of the main distinctions that set intelligent systems apart from other types of systems, is with regards to how uncertainty is handled. As the RfGA framework is associated to uncertain fuzzy membership values, uncertainty is often a common issue when considering fuzzy memberships, and therefore can be a problem in the application of fuzzy sets. As it has already been stated, there have been numerous extensions to that of a type-1 fuzzy set. The notion of a type-2 fuzzy set is favourable, if not for the high cost burden. A type-2 approach does indeed have a very strong theoretical capacity with regards to representing the uncertainty it is capturing, but there are significant concerns when considering its highly subjective type-2 membership. The computational burden is also a limiting factor in a generalised type- 2 approach, hence why the interval-valued type-2 fuzzy set is often adopted. As Theorem 2 and Proof 2 have shown, when connecting an R-fuzzy set and significance measure pairing to that of a type-2 fuzzy set, one is presented with a novel methodology to solve the problem of generalised type-2 fuzzy sets in application. This was achieved by ultimately replacing the subjective membership set with that of a collection comprised of discrete objective type-1 fuzzy membership values, of an R-fuzzy membership set. By presenting it in this way, the additional precision one can attribute to a type-2 approach is preserved, however, the difficulty associated to it is heavily reduced as too is the difficulty in defying a generalised type-2 fuzzy membership.

The main contributions of this publication and proposed framework can be summarised as follows; it is a simplistic configuration of 3 key components - R-fuzzy sets; the significance measure; and the absolute degree of grey incidence. This allows for a greater detail of information to be garnered. The captured uncertainty suffers no loss of information unlike that of an interval approach, for every contained uncertain fuzzy membership value belonging to the membership set can be uniquely distinguishable. Each captured value can then be extrapolated based on the conditional probability of the population to determine how favourable any specific membership value is, relative to the R-fuzzy set that as being modelled. This paper puts forward the framework in its entirety, making use of grey relational analysis which provides for an additional level of post-analysis. The framework when considering only an R-fuzzy set and significance pairing as already been shown to provide a means to offer an alternative to a generalised type-2 fuzzy approach (Khuman et al., 2016a). The addition of grey analysis offers an added advantage in providing more detail. The incorporation of grey theory into uncertainty frameworks has now started to become more noticeable (Salmeron and Gutierrez, 2012), (Memon et al., 2015), (Tseng, 2010), but this proposed framework adapts a grey component and deploys it in an unconventional, yet effective manner. The novelty of the framework allows for one to fully capture all the nuances and individualities of a population without a single loss of information. That snapshot in time can tell an awful lot with regards to perceived perception. Potentially, one can use that snapshot to predict how an observation could be perceived in future events. If 
one can forecast the perception ahead of time, one can improve drastically on efficacy and efficiency. Such a framework has a strong applicability with regards to expert and intelligent systems in allowing for more detailed inference to be utilised and acted upon. If the formalisation of such systems are based on the inference provided by this framework, one can anticipate more accurate and effective systems as a result.

The R-fuzzy and grey analysis framework provides one with the capabilities to explore a multitude of different avenues. It is envisioned that the framework can be deployed in any setting requiring one to collect the perceptions concerning any initial observation. Using the collected metrics derived when utilising the absolute degree of grey incidence, one can capture the perception of an individual, or cohort indicative of a demographic, then one can use this information to aid and facilitate the creation of better perceived observations, whether they are visual, audio, and so on. Knowing how a demographic currently perceives an observation, serves a great purpose in anticipating how they could possibly perceive the same observation, or variance of that observation in the future. Providing this information for an expert or an intelligent system will allow for a system with greater detailed resolution based on higher derived inference.

Another possible direction for future research is to investigate if the framework can indeed be used to detect underlying issues. If a member of the populous was to return a response which equated to a specifically derived membership value, then one could assume that the individual may be susceptible to a particular disability, whether it be visual or audio for example. The information collected and presented by the framework could aid in the creation of better configured expert and intelligent systems for detection purposes.

Given how robust and versatile the current framework is, investigating its most beneficial application domain should be further researched. It does seem to show merit when visual observations are the subject. When R-fuzzy sets were originally proposed in Yang and Hinde (2010), the examples that were demonstrated were audio based, therefore, the RfGA framework would also show good applicability in this regard. The authors would like to investigate how the perception of understanding could be modelled. Consider if a written policy was presented to a population and the understanding of that policy was questioned. The results could determine if certain cohorts or demographics found such a policy more understandable than others, in which case reforms could be made to target specific clusters.

One of the proposed areas of future research is to explore the applicability of the framework with regards to higher dimensional problems. In its current configuration, subjective based domains associated to 3 dimensions are preferable. Similar to type-n fuzzy sets, we believe that a possible type-n framework would also be a possibility, this will be the basis of a follow-up publication. If it can be proved that the framework can be modified to cater for higher dimension, this would improve on the applicability and reach.

As of yet the existence of R-fuzzy sets and the R-fuzzy and grey analysis framework and its applicability are not widely known. To that end, one hopes a clearer picture has been painted and more interest garnered. 


\section{Conflict of Interest Statement}

The authors declare that there are no conflicts of interest regarding the publication of this paper.

\section{References}

\section{References}

Amirkhan, M., Didehkhani, H., Khalili-Damghani, K., and Hafezalkotob, A. (2018). Mixed uncertainties in data envelopment analysis: A fuzzy-robust approach. Expert Systems with Applications, 103:218 - 237.

Atanassov, K. T. (1986). Intuitionistic fuzzy sets. Fuzzy Sets and Systems, 20(1):87 - 96.

Bodjanova, S. (2007). Granulation of a fuzzy set: Nonspecificity. Information Sciences, 177(20):4430 - 4444.

de S. Sampaio Filho, A. C., Vellasco, M. M., and Tanscheit, R. (2018). A unified solution in fuzzy capital budgeting. Expert Systems with Applications, 98:27 - 42 .

Deng, T., Chen, Y., Xu, W., and Dai, Q. (2007). A novel approach to fuzzy rough sets based on a fuzzy covering. Information Sciences, 177(11):2308 - 2326.

Dubois, D. and Prade, H. (1990). Rough fuzzy sets and fuzzy rough sets*. International Journal of General Systems, 17(23):191-209.

Dubois, D. J. (1980). Fuzzy sets and systems: theory and applications, volume 144. Academic press.

Dwivedi, G., Srivastava, R. K., and Srivastava, S. K. (2018). A generalised fuzzy topsis with improved closeness coefficient. Expert Systems with Applications, 96:185 - 195.

Huynh, V.-N. and Nakamori, Y. (2005). A roughness measure for fuzzy sets. Information Sciences, 173(1-3):255 - 275.

Jensen, R. and Shen, Q. (2008). Computational intelligence and feature selection: rough and fuzzy approaches, volume 8. John Wiley \& Sons.

Jensen, R. and Shen, Q. (2009). New approaches to fuzzy-rough feature selection. Fuzzy Systems, IEEE Transactions on, $17(4): 824-838$.

Khuman, A., Yang, Y., and John, R. (2016a). Quantification of r-fuzzy sets. Expert Systems with Applications, 55:374 - 387.

Khuman, A. S. (2016). The quantification of perception based uncertainty using R-fuzzy sets and grey analysis. PhD thesis, De Montfort University, UK.

Khuman, A. S., Yang, Y., and John, R. (2015a). A significance measure for r-fuzzy sets. In Fuzzy Systems (FUZZ-IEEE), 2015 IEEE International Conference on, pages 1-6.

Khuman, A. S., Yang, Y., and John, R. (2016b). R-fuzzy sets and grey system theory. In 2016 IEEE International Conference on Systems, Man, and Cybernetics.

Khuman, A. S., Yang, Y., and Liu, S. (2015b). Grey relational analysis and natural language processing. In 2015 IEEE International Conference on Grey Systems and Intelligent Services (GSIS), pages 107-112.

Liu, S. and Lin, Y. (2006). Grey Information: Theory and Applications. Advanced Information and Knowledge Processing. Springer-Verlag London, 1 edition.

Melin, P. and Castillo, O. (2014). A review on type-2 fuzzy logic applications in clustering, classification and pattern recognition. Applied Soft Computing, 21(0):568 - 577.

Melin, P., Miramontes, I., and Prado-Arechiga, G. (2018). A hybrid model based on modular neural networks and fuzzy systems for classification of blood pressure and hypertension risk diagnosis. Expert Systems with Applications, 107:146 - 164.

Memon, M. S., Lee, Y. H., and Mari, S. I. (2015). Group multi-criteria supplier selection using combined grey systems theory and uncertainty theory. Expert Systems with Applications, 42(21):7951 - 7959.

Mendel, J. and John, R. (2002). Type-2 fuzzy sets made simple. Fuzzy Systems, IEEE Transactions on, 10(2):117-127. 
Muhanna, R. L. and Mullen, R. L. (2001). Uncertainty in mechanics problems\&\#x2014;interval\&\#x2013;based approach. Journal of Engineering Mechanics, 127(6):557-566.

Nanda, S. and Majumdar, S. (1992). Fuzzy rough sets. Fuzzy Sets and Systems, 45(2):157 - 160.

Pawlak, Z. (1982). Rough sets. International Journal of Computer $\& 3$ Information Sciences, 11(5):341-356.

Pawlak, Z. (1998). Rough set theory and its applications to data analysis. Cybernetics \& Systems, 29(7):661-688.

Pawlak, Z. (2002). Rough sets and intelligent data analysis. Information Sciences, 147(1-4):1 - 12.

Pawlak, Z. and Skowron, A. (2007). Rough sets: Some extensions. Information Sciences, 177(1):28 - 40. Zdzis?aw Pawlak life and work (1926-2006).

Pedrycz, W. (1998). Shadowed sets: representing and processing fuzzy sets. Systems, Man, and Cybernetics, Part B: Cybernetics, IEEE Transactions on, 28(1):103-109.

Radzikowska, A. M. and Kerre, E. E. (2002). A comparative study of fuzzy rough sets. Fuzzy Sets and Systems, 126(2):137 155.

Salmeron, J. L. (2010). Modelling grey uncertainty with fuzzy grey cognitive maps. Expert Systems with Applications, $37(12): 7581-7588$.

Salmeron, J. L. and Gutierrez, E. (2012). Fuzzy grey cognitive maps in reliability engineering. Applied Soft Computing, 12(12):3818 - 3824. Theoretical issues and advanced applications on Fuzzy Cognitive Maps.

Sambuc, R. (1975). Fonctions $\Phi$ foues. Application a l'aide au Diagnostic en Patholologie Thyroidienne. PhD thesis, Univ. Marseille, France.

Shafer, G. (1976). A Mathematical Theory of Evidence. Princeton University Press, Princeton.

Sifeng, L., Forrest, J., and Yingjie, Y. (2011). A brief introduction to grey systems theory. In Grey Systems and Intelligent Services (GSIS), 2011 IEEE International Conference on, pages 1-9.

Sun, B., Ma, W., and Chen, D. (2014). Rough approximation of a fuzzy concept on a hybrid attribute information system and its uncertainty measure. Information Sciences, 284(0):60 - 80. Special issue on Cloud-assisted Wireless Body Area Networks.

Tiwari, A. K., Shreevastava, S., Som, T., and Shukla, K. (2018). Tolerance-based intuitionistic fuzzy-rough set approach for attribute reduction. Expert Systems with Applications, 101:205 - 212.

Tseng, M.-L. (2010). Using linguistic preferences and grey relational analysis to evaluate the environmental knowledge management capacity. Expert Systems with Applications, 37(1):70 - 81.

Wang, C. and Matthies, H. G. (2019). Hybrid evidence-and-fuzzy uncertainty propagation under a dual-level analysis framework. Fuzzy Sets and Systems, 367:51 - 67. Theme: Uncertainty Management.

Wu, W.-Z., Mi, J.-S., and Zhang, W.-X. (2003). Generalized fuzzy rough sets. Information Sciences, $151(0): 263$ - 282.

$\mathrm{Xu}, \mathrm{W}$. , Liu, Y., and Sun, W. (2012). Intuitionistic fuzzy rough sets model based on ( $\theta$, $\phi)$-operators. In Fuzzy Systems and Knowledge Discovery (FSKD), 2012 9th International Conference on, pages 234-238. IEEE.

Yang, Y. and Hinde, C. (2010). A new extension of fuzzy sets using rough sets: R-fuzzy sets. Information Sciences, 180(3):354 -365 .

Yao, Y. (1996). Two views of the theory of rough sets in finite universes. International Journal of Approximate Reasoning, 15(4):291 - 317. Rough Sets.

Zadeh, L. A. (1965). Fuzzy sets. Information and control, 8(3):338-353.

Zeng, A., Li, T., Liu, D., Zhang, J., and Chen, H. (2015). A fuzzy rough set approach for incremental feature selection on hybrid information systems. Fuzzy Sets and Systems, 258(0):39 - 60. Special issue: Uncertainty in Learning from Big Data. 